\title{
Solvability of a system of higher order nonlinear difference equations
}

\author{
Merve Kara ${ }^{1}$ (D), Yasin Yazlik*2 (D), Durhasan Turgut Tollu ${ }^{3}$ \\ ${ }^{1}$ Ortaköy Vocational School of Higher Education, Aksaray University, 68400 Aksaray, Turkey \\ ${ }^{2}$ Department of Mathematics, Faculty of Science and Art, Nevsehir Hacı Bektaş Veli University, \\ 50300 Nevsehir, Turkey \\ ${ }^{3}$ Department of Mathematics-Computer Sciences, Faculty of Science, Necmettin Erbakan University, \\ 42090 Konya, Turkey
}

\begin{abstract}
In this paper we show that the system of difference equations

$$
x_{n}=a y_{n-k}+\frac{d y_{n-k} x_{n-(k+l)}}{b x_{n-(k+l)}+c y_{n-l}}, y_{n}=\alpha x_{n-k}+\frac{\delta x_{n-k} y_{n-(k+l)}}{\beta y_{n-(k+l)}+\gamma x_{n-l}},
$$

where $n \in \mathbb{N}_{0}, k$ and $l$ are positive integers, the parameters $a, b, c, d, \alpha, \beta, \gamma, \delta$ are real numbers and the initial values $x_{-j}, y_{-j}, j=\overline{1, k+l}$, are real numbers, can be solved in the closed form. We also determine the asymptotic behavior of solutions for the case $l=1$ and describe the forbidden set of the initial values using the obtained formulas. Our obtained results significantly extend and develop some recent results in the literature.
\end{abstract}

Mathematics Subject Classification (2010). 39A10, 39A20, 39A23, 11 B39

Keywords. system of difference equations, asymptotic behavior, Fibonacci sequence, forbidden set

\section{Introduction and preliminaries}

For the past two decades there has been an intense interest in nonlinear difference equations, see $[2,5-7,18,36,37,40,42,44]$. In the meantime, the two-dimensional or threedimensional systems of these equations have become the center of interest of researchers. See, for example, $[9,16,17,22,25,34,38,43,45,46,48]$. Theoretically, it is very important to characterize the behavior of the solutions of these equations and systems. Although many methods are proposed by researchers, the most basic method to do this is to find a closed formula of the solution of the equation or system and analyze it. In [21], McGrath and Teixeira studied the equation

$$
x_{n+1}=\frac{a x_{n-1}+b x_{n}}{c x_{n-1}+d x_{n}} x_{n}, n \in \mathbb{N}_{0},
$$

\footnotetext{
*Corresponding Author.

Email addresses: mervekara@aksaray.edu.tr (M. Kara), yyazlik@nevsehir.edu.tr (Y. Yazlik), dttollu@konya.edu.tr (D.T. Tollu)

Received: 25.10.2018; Accepted: 22.11.2019
} 
where the parameters $a, b, c, d$, and the initial values are real numbers. The authors solved Eq. (1.1) and investigated the existence and behavior of the solution of Eq. (1.1) by using some known results. In [39], Tollu et al. considered the following difference equations

$$
x_{n}=\alpha x_{n-k}+\frac{\delta x_{n-k} x_{n-(k+l)}}{\beta x_{n-(k+l)}+\gamma x_{n-l}}, n \in \mathbb{N}_{0},
$$

where $k$ and $l$ are fixed natural numbers, $\alpha, \beta, \gamma, \delta \in \mathbb{R}$, and the initial values $x_{-i}$, $i=\overline{1, k+l}$, are real numbers. The authors showed that Eq. (1.2) is solvable in closed form and presented formulas for the solutions. They also studied the long-term behavior of the solutions of Eq. (1.2). Some particular cases of the extension of Eq. (1.2) have been studied recently in papers [11-14,33]. More precisely, Eq. (1.2) in the case $\alpha=0, \delta=$ $1, \beta=1, \gamma= \pm 1$ was studied in $[11,12]$ and in [33] the case $\alpha=0, \delta=1$ was studied, while in $[13,14]$ the case $k=1, l=1$ and $k=2, l=2$ were studied. Eq. (1.1) and Eq. (1.2) actually are particular cases of the higher-order difference equation

$$
\frac{x_{n}}{x_{n-k}}=f\left(\frac{x_{n-l}}{x_{n-k-l}}\right), n \in \mathbb{N}_{0},
$$

where $k$ and $l$ are fixed natural numbers. If the equation $y_{n}=f\left(y_{n-l}\right), n \in \mathbb{N}_{0}$, is a solvable type, then Eq. (1.3) is solvable, too.

On the other hand, in [41], Eq. (1.2) in the case $\alpha=0, \delta=1, \beta= \pm 1, \gamma= \pm 1$ was extended to the following two-dimensional system of difference equation

$$
x_{n+1}=\frac{y_{n} x_{n-1}}{ \pm x_{n-1} \pm y_{n}}, y_{n+1}=\frac{x_{n} y_{n-1}}{ \pm y_{n-1} \pm x_{n}}, n \in \mathbb{N}_{0},
$$

with real nonzero initial values $x_{-i}, y_{-i}$, for $i=0,1$, such that some of their solutions are associated to Fibonacci numbers and some of their solution formulas were proved by induction. However, the formulas have not been confirmed by some theoretical explanations.

In this paper, we give some theoretical explanations for the formulas of solutions of the difference equations system given in (1.4). Moreover, we show that the following more general difference equation system

$$
x_{n}=a y_{n-k}+\frac{d y_{n-k} x_{n-(k+l)}}{b x_{n-(k+l)}+c y_{n-l}}, y_{n}=\alpha x_{n-k}+\frac{\delta x_{n-k} y_{n-(k+l)}}{\beta y_{n-(k+l)}+\gamma x_{n-l}}, n \in \mathbb{N}_{0},
$$

where $k$ and $l$ are positive integers, $a, b, c, d, \alpha, \beta, \gamma, \delta \in \mathbb{R}$, and the initial values $x_{-j}$, $y_{-j}, j=\overline{1, k+l}$, are real numbers, can be solved closed form. Also, we investigate some particular cases of system (1.5) and give a study of the long-term behavior of its solutions for the case $l=1$. Finally, we also give natural explanation for the formulas presented in [41].

For more works on the topic, see, for example, $[3,4,8,10,23,24,26-32,35,47]$ and the references therein. Also, see the books $[1,15,20]$.

Now, we should recall that the Fibonacci sequence $\left\{F_{n}\right\}_{n=0}^{\infty}$ is defined by

$$
F_{n+2}=F_{n+1}+F_{n}, n \in \mathbb{N}_{0},
$$

with the initial values $F_{0}$ and $F_{1}$. Considering [19], the characteristic equation of (1.6) can be clearly obtained as of the form $x^{2}-x-1=0$ having the roots $\alpha=\frac{1+\sqrt{5}}{2}$ and $\beta=\frac{1-\sqrt{5}}{2}$. Thus, the Binet's Formula for Fibonacci sequence, $F_{n}=\frac{\alpha^{n}-\beta^{n}}{\alpha-\beta}$, can be thought as a solution of Fibonacci sequence. Also, it is obtained to extend negatively subscripted Fibonacci sequence as

$$
F_{-n}=F_{-n+2}-F_{-n+1}=(-1)^{n+1} F_{n}, n \in \mathbb{N}_{0} .
$$




\section{Solvability of system (1.5)}

In this section we show that the system (1.5) is solvable in closed form. First, we write the system as follows:

$$
\frac{x_{n}}{y_{n-k}}=\frac{a c \frac{y_{n-l}}{x_{n-(k+l)}}+a b+d}{c \frac{y_{n-l}}{x_{n-(k+l)}}+b}, \frac{y_{n}}{x_{n-k}}=\frac{\alpha \gamma \frac{x_{n-l}}{y_{n-(k+l)}}+\alpha \beta+\delta}{\gamma \frac{x_{n-l}}{y_{n-(k+l)}}+\beta}, n \in \mathbb{N}_{0} .
$$

Putting

$$
u_{n}=\frac{x_{n}}{y_{n-k}}, v_{n}=\frac{y_{n}}{x_{n-k}}, n \geq-l,
$$

in the last expressions, we get the system of equations

$$
u_{n}=\frac{a c v_{n-l}+a b+d}{c v_{n-l}+b}, v_{n}=\frac{\alpha \gamma u_{n-l}+\alpha \beta+\delta}{\gamma u_{n-l}+\beta}, n \in \mathbb{N}_{0},
$$

where the parameters $a, b, c, d, \alpha, \beta, \gamma, \delta$, in the new variables $u_{n}$ and $v_{n}$. By using the first equation of (2.2) in its second equation and its second equation in its first equation, we obtain the independent equations

$$
u_{n}=\frac{(a c \alpha \gamma+a b \gamma+d \gamma) u_{n-2 l}+a c \alpha \beta+a c \delta+a b \beta+d \beta}{(c \alpha \gamma+b \gamma) u_{n-2 l}+c \alpha \beta+c \delta+b \beta}, n \geq l,
$$

and

$$
v_{n}=\frac{(a c \alpha \gamma+c \alpha \beta+c \delta) v_{n-2 l}+a b \alpha \gamma+\alpha \gamma d+\alpha \beta b+\delta b}{(\gamma a c+\beta c) v_{n-2 l}+\gamma a b+\gamma d+b \beta}, n \geq l .
$$

If we apply the decomposition of indexes $n \rightarrow 2 l m+i$, for $m \geq-1$ and $i \in\{l, l+1, \ldots, 3 l-$ $1\}$, to (2.3) and (2.4), they become

$$
\begin{aligned}
& u_{2 l m+i}=\frac{(a c \alpha \gamma+a b \gamma+d \gamma) u_{2 l(m-1)+i}+a c \alpha \beta+a c \delta+a b \beta+d \beta}{(c \alpha \gamma+b \gamma) u_{2 l(m-1)+i}+c \alpha \beta+c \delta+b \beta}, m \in \mathbb{N}_{0}, \\
& v_{2 l m+i}=\frac{(a c \alpha \gamma+c \alpha \beta+c \delta) v_{2 l(m-1)+i}+a b \alpha \gamma+\alpha \gamma d+\alpha \beta b+\delta b}{(\gamma a c+\beta c) v_{2 l(m-1)+i}+\gamma a b+\gamma d+b \beta}, m \in \mathbb{N}_{0} .
\end{aligned}
$$

Let $u_{m}^{(i)}=u_{2 l m+i}, v_{m}^{(i)}=v_{2 l m+i}$, for some $m \geq-1$ and $i \in\{l, l+1, \ldots, 3 l-1\}$. Then equations in (2.5)-(2.6) can be written as the following:

$$
\begin{aligned}
& u_{m}^{(i)}=\frac{(a c \alpha \gamma+a b \gamma+d \gamma) u_{m-1}^{(i)}+a c \alpha \beta+a c \delta+a b \beta+d \beta}{(c \alpha \gamma+b \gamma) u_{m-1}^{(i)}+c \alpha \beta+c \delta+b \beta}, m \in \mathbb{N}_{0}, \\
& v_{m}^{(i)}=\frac{(a c \alpha \gamma+c \alpha \beta+c \delta) v_{m-1}^{(i)}+a b \alpha \gamma+\alpha \gamma d+\alpha \beta b+\delta b}{(\gamma a c+\beta c) v_{m-1}^{(i)}+\gamma a b+\gamma d+b \beta}, m \in \mathbb{N}_{0},
\end{aligned}
$$

which is essentially in the form of Riccati difference equation. If we use the change of variables

$$
u_{m}^{(i)}=\frac{a c \alpha \gamma+a b \gamma+d \gamma+c \alpha \beta+c \delta+b \beta}{c \alpha \gamma+b \gamma} r_{m}-\frac{c \alpha \beta+c \delta+b \beta}{c \alpha \gamma+b \gamma}, m \geq-1,
$$

where $c \alpha \gamma+b \gamma \neq 0$, in Eq. (2.7) and the change of variables

$$
v_{m}^{(i)}=\frac{a c \alpha \gamma+a b \gamma+d \gamma+c \alpha \beta+c \delta+b \beta}{\gamma a c+\beta c} s_{m}-\frac{\gamma a b+\gamma d+b \beta}{\gamma a c+\beta c}, m \geq-1,
$$

where $\gamma a c+\beta c \neq 0$, in Eq. (2.8), then (2.7) and (2.8) are transformed into the following equations:

$$
r_{m}=1-\frac{R}{r_{m-1}}, s_{m}=1-\frac{R}{s_{m-1}}, m \in \mathbb{N}_{0},
$$


where $a c \alpha \gamma+a b \gamma+d \gamma+c \alpha \beta+c \delta+b \beta \neq 0$ and $R=\frac{c d \gamma \delta}{(a c \alpha \gamma+a b \gamma+d \gamma+c \alpha \beta+c \delta+b \beta)^{2}}$, respectively. The equations in (2.11) can be transformed into the following equations:

$$
\begin{aligned}
& z_{m+1}=z_{m}-R z_{m-1}, m \in \mathbb{N}_{0}, \\
& \widehat{z}_{m+1}=\widehat{z}_{m}-R \widehat{z}_{m-1}, m \in \mathbb{N}_{0},
\end{aligned}
$$

by means of the changes of variables $r_{m}=\frac{z_{m+1}}{z_{m}}$ with the initial values $z_{-1}=1$ and $z_{0}=r_{-1}$ and $s_{m}=\frac{\widehat{z}_{m+1}}{\widehat{z}_{m}}$ with the initial values $\widehat{z}_{-1}=1$ and $\widehat{z}_{0}=s_{-1}$, respectively. (2.12) and (2.13) are in the same form and have the characteristic equation $\lambda^{2}-\lambda+R=0$. If $\lambda_{1}$ and $\lambda_{2}$ are the roots of the characteristic equations, namely $\lambda_{1}=\frac{1+\sqrt{1-4 R}}{2}$ and $\lambda_{2}=\frac{1-\sqrt{1-4 R}}{2}$, the general solutions of equations in (2.12) and (2.13) are

$$
\begin{aligned}
& z_{m}=\left\{\begin{array}{ll}
\frac{\left(\lambda_{1} r_{-1}-R\right) \lambda_{1}^{m}-\left(\lambda_{2} r_{-1}-R\right) \lambda_{2}^{m}}{\lambda_{1}-\lambda_{2}} & \text { if } R \neq \frac{1}{4}, \\
\left(\frac{2 r_{-1}+\left(2 r_{-1}-1\right) m}{2}\right)\left(\frac{1}{2}\right)^{m} & \text { if } R=\frac{1}{4},
\end{array},\right. \\
& \widehat{z}_{m}= \begin{cases}\frac{\left(\lambda_{1} s_{-1}-R\right) \lambda_{1}^{m}-\left(\lambda_{2} s_{-1}-R\right) \lambda_{2}^{m}}{\lambda_{1}-\lambda_{2}} & \text { if } R \neq \frac{1}{4}, \\
\left(\frac{2 s_{-1}+\left(2 s_{-1}-1\right) m}{2}\right)\left(\frac{1}{2}\right)^{m} & \text { if } R=\frac{1}{4},\end{cases}
\end{aligned}
$$

where $R=\frac{c d \gamma \delta}{(a c \alpha \gamma+a b \gamma+d \gamma+c \alpha \beta+c \delta+b \beta)^{2}}$. By substituting (2.14) into $r_{m}=\frac{z_{m+1}}{z_{m}}$ and (2.15) into $s_{m}=\frac{\widehat{z}_{m+1}}{\widehat{z}_{m}}$, we have

$$
\begin{aligned}
& r_{m}= \begin{cases}\frac{\left(\lambda_{1} r_{-1}-R\right) \lambda_{1}^{m+1}-\left(\lambda_{2} r_{-1}-R\right) \lambda_{2}^{m+1}}{\left(\lambda_{1} r_{-1}-R\right) \lambda_{1}^{m}-\left(\lambda_{2} r_{-1}-R\right) \lambda_{2}^{m}} & \text { if } R \neq \frac{1}{4}, m \geq-1, \\
\frac{2 r_{-1}+\left(2 r_{-1}-1\right)(m+1)}{4 r_{-1}+\left(4 r_{-1}-2\right) m} & \text { if } R=\frac{1}{4},\end{cases} \\
& s_{m}= \begin{cases}\frac{\left(\lambda_{1} s_{-1}-R\right) \lambda_{1}^{m+1}-\left(\lambda_{2} s_{-1}-R\right) \lambda_{2}^{m+1}}{\left(\lambda_{1} s_{-1}-R\right) \lambda_{1}^{m}-\left(\lambda_{2} s_{-1}-R\right) \lambda_{2}^{m}} & \text { if } R \neq \frac{1}{4}, m \geq-1, \\
\frac{2 s_{-1}+\left(2 s_{-1}-1\right)(m+1)}{4 s_{-1}+\left(4 s_{-1}-2\right) m} & \text { if } R=\frac{1}{4},\end{cases}
\end{aligned}
$$

respectively. Consequently, we get

$$
u_{m}^{(i)}= \begin{cases}\frac{A}{B_{1}} \frac{\left(\lambda_{1} B_{1} u_{-1}^{(i)}+\lambda_{1} C_{1}-R A\right) \lambda_{1}^{m+1}-\left(\lambda_{2} B_{1} u_{-1}^{(i)}+\lambda_{2} C_{1}-R A\right) \lambda_{2}^{m+1}}{\left(\lambda_{1} B_{1} u_{-1}^{(i)}+\lambda_{1} C_{1}-R A\right) \lambda_{1}^{m}-\left(\lambda_{2} B_{1} u_{-1}^{(i)}+\lambda_{2} C_{1}-R A\right) \lambda_{2}^{m}}-\frac{C_{1}}{B_{1}} & \text { if } R \neq \frac{1}{4}, \\ \frac{A}{B_{1}}\left(\frac{2 B_{1} u_{-1}^{(i)}+2 C_{1}+\left(2 B_{1} u_{-1}^{(i)}+2 C_{1}-A\right)(m+1)}{4 B_{1} u_{-1}^{(i)}+4 C_{1}+\left(4 B_{1} u_{-1}^{(i)}+4 C_{1}-2 A\right) m}\right)-\frac{C_{1}}{B_{1}} & \text { if } R=\frac{1}{4},\end{cases}
$$

and

$$
v_{m}^{(i)}= \begin{cases}\frac{A}{B_{2}} \frac{\left(\lambda_{1} B_{2} v_{-1}^{(i)}+\lambda_{1} C_{2}-R A\right) \lambda_{1}^{m+1}-\left(\lambda_{2} B_{2} v_{-1}^{(i)}+\lambda_{2} C_{2}-R A\right) \lambda_{2}^{m+1}}{\left(\lambda_{1} B_{2} v_{-1}^{(i)}+\lambda_{1} C_{2}-R A\right) \lambda_{1}^{m}-\left(\lambda_{2} B_{2} v_{-1}^{(i)}+\lambda_{2} C_{2}-R A\right) \lambda_{2}^{m}}-\frac{C_{2}}{B_{2}} & \text { if } R \neq \frac{1}{4}, \\ \frac{A}{B_{2}}\left(\frac{2 B_{2} v_{-1}^{(i)}+2 C_{2}+\left(2 B_{2} v_{-1}^{(i)}+2 C_{2}-A\right)(m+1)}{4 B_{2} v_{-1}^{(i)}+4 C_{2}+\left(4 B_{2} v_{-1}^{(i)}+4 C_{2}-2 A\right) m}\right)-\frac{C_{2}}{B_{2}} & \text { if } R=\frac{1}{4},\end{cases}
$$

and so

$$
u_{2 l m+i}= \begin{cases}\frac{A}{B_{1}} \frac{G_{1} \lambda_{1}^{m+1}-G_{2} \lambda_{2}^{m+1}}{G_{1} \lambda_{1}^{m}-G_{2} \lambda_{2}^{m}}-\frac{C_{1}}{B_{1}} & \text { if } R \neq \frac{1}{4}, \\ \frac{A}{B_{1}}\left(\frac{H_{1}+\left(H_{1}-A\right)(m+1)}{2 H_{1}+\left(2 H_{1}-2 A\right) m}\right)-\frac{C_{1}}{B_{1}} & \text { if } R=\frac{1}{4},\end{cases}
$$

and

$$
v_{2 l m+i}= \begin{cases}\frac{A}{B_{2}} \frac{K_{1} \lambda_{1}^{m+1}-K_{2} \lambda_{2}^{m+1}}{K_{1} \lambda_{1}^{m}-K_{2} \lambda_{2}^{m}}-\frac{C_{2}}{B_{2}} & \text { if } R \neq \frac{1}{4}, \\ \frac{A}{B_{2}}\left(\frac{H_{2}+\left(H_{2}-A\right)(m+1)}{2 H_{2}+\left(2 H_{2}-2 A\right) m}\right)-\frac{C_{2}}{B_{2}} & \text { if } R=\frac{1}{4},\end{cases}
$$

where $A=a c \alpha \gamma+a b \gamma+d \gamma+c \alpha \beta+c \delta+b \beta, B_{1}=c \alpha \gamma+b \gamma, C_{1}=c \alpha \beta+c \delta+b \beta, B_{2}=\gamma a c+\beta c$, $C_{2}=\gamma a b+\gamma d+\beta b, G_{1}=\lambda_{1} B_{1} \frac{x_{i-2 l}}{y_{i-2 l-k}}+\lambda_{1} C_{1}-R A, G_{2}=\lambda_{2} B_{1} \frac{x_{i-2 l}}{y_{i-2 l-k}}+\lambda_{2} C_{1}-R A$, 
$K_{1}=\lambda_{1} B_{2} \frac{y_{i-2 l}}{x_{i-2 l-k}}+\lambda_{1} C_{2}-R A, K_{2}=\lambda_{2} B_{2} \frac{y_{i-2 l}}{x_{i-2 l-k}}+\lambda_{2} C_{2}-R A, H_{1}=2 B_{1} \frac{x_{i-2 l}}{y_{i-2 l-k}}+2 C_{1}$, $H_{2}=2 B_{2} \frac{y_{i-2 l}}{x_{i-2 l-k}}+2 C_{2}$ for $i \in\{l, l+1 \ldots, 3 l-1\}$. From (2.1) we have that

$$
\begin{aligned}
x_{n}=u_{n} y_{n-k} & =u_{n} v_{n-k} x_{n-2 k}, y_{n}=v_{n} x_{n-k}=v_{n} u_{n-k} y_{n-2 k}, n \geq k-l, \\
x_{2 k m+i} & =u_{2 k m+i} y_{2 k m+i-k}=u_{2 k m+i} v_{2 k m+i-k} x_{2 k(m-1)+i}, m \in \mathbb{N}_{0}, \\
y_{2 k m+i} & =v_{2 k m+i} x_{2 k m+i-k}=v_{2 k m+i} u_{2 k m+i-k} y_{2 k(m-1)+i}, m \in \mathbb{N}_{0},
\end{aligned}
$$

from which it follows that

$$
x_{2 k m+i}=x_{i-2 k} \prod_{j=0}^{m} u_{2 k j+i} v_{2 k j+i-k},
$$

and

$$
y_{2 k m+i}=y_{i-2 k} \prod_{j=0}^{m} v_{2 k j+i} u_{2 k j+i-k},
$$

for every $m \in \mathbb{N}_{0}$ and $i=\overline{k-l, 3 k-l-1}$. Since every non-negative integer can be written in the form $l m_{1}+j$, where $m_{1} \in \mathbb{N}_{0}$ and $j \in\{0,1, \ldots, l-1\}$, we get that

$$
\begin{aligned}
& x_{2 k l m_{1}+2 k j+i}=x_{2 k j+i-2 k} \prod_{s=0}^{l m_{1}} u_{2 k s+2 k j+i} v_{2 k s+2 k j+i-k}, \quad m_{1} \in \mathbb{N}_{0}, \\
& y_{2 k l m_{1}+2 k j+i}=y_{2 k j+i-2 k} \prod_{s=0}^{l m_{1}} v_{2 k s+2 k j+i} u_{2 k s+2 k j+i-k}, \quad m_{1} \in \mathbb{N}_{0},
\end{aligned}
$$

where $j \in\{0,1, \ldots, l-1\}$ and $i=\overline{k-l, 3 k-l-1}$.

By the following theorem, we characterize the forbidden set of the initial values for the system (1.5).

Theorem 2.1. The forbidden set of the initial values for system (1.5) is the union of two sets

$$
\left\{\vec{X}: x_{-j}=0 \text { or } y_{-j}=0, j=\overline{1, k}\right\}
$$

and

$$
\begin{aligned}
\bigcup_{m \in \mathbb{N}_{0}} \bigcup_{j=1}^{k+l} & \left\{\vec{X}: \frac{y_{j-l}}{x_{j-k-l}}=(f \circ g)^{-m}\left(-\frac{b}{c}\right) \text { or } \frac{x_{j-l}}{y_{j-k-l}}=(g \circ f)^{-m}\left(K_{1}\right)\right. \\
& \text { or } \left.(g \circ f)^{-m}\left(-\frac{\beta}{\gamma}\right) \text { or }(f \circ g)^{-m}\left(K_{2}\right)\right\},
\end{aligned}
$$

where $\vec{X}=\left(x_{-k-l}, x_{-k-l+1}, \ldots, x_{-1}, y_{-k-l}, y_{-k-l+1}, \ldots, y_{-1}\right), K_{1}=-\frac{b \beta+c \alpha \beta+c \delta}{b \gamma+c \alpha \gamma}$, $K_{2}=-\frac{b \beta+a b \gamma+d \gamma}{c \beta+a c \gamma}$.

Proof. Let $\left(x_{n}, y_{n}\right)_{n>-k-l}$ be a solution of the system (1.5). If $x_{-j}=0$ or $y_{-j}=0$ for some $j=\overline{1, k}$, then $x_{n}$ cannot be calculated after a term $y_{n_{0}}, n_{0} \in \mathbb{N}_{0}$. For example, if $x_{-k}=0$, then $y_{0}=0$, and so $x_{l}$ cannot be calculated. For the dual of this case, the result is same, too. That is, if $y_{-k}=0$, then $x_{0}=0$, and so $y_{l}$ cannot be calculated. For the other initial values, the case is not same. Because, if $x_{-j}=0$ or $y_{-j}=0$ for some $j=\overline{k+1, k+l}$, then $x_{n} \neq 0, y_{n} \neq 0$ for $n \geq 0$. So, we incorporate the set

$$
\left\{\vec{X}: x_{-j}=0 \text { or } y_{-j}=0, j=\overline{1, k}\right\}
$$

in the forbidden set. Now, we suppose that $x_{n} \neq 0$ and $y_{n} \neq 0$. The solution $\left(x_{n}, y_{n}\right)_{n \geq-k-l}$ of the system (1.5) is not defined if and only if $b x_{n-(k+l)}+c y_{n-l}=0$ and $\beta y_{n-(k+l)}+\gamma x_{n-l}=$ 
0 which correspond to the statements $\frac{y_{n-l}}{x_{n-k-l}}=-\frac{b}{c}$ and $\frac{x_{n-l}}{y_{n-k-l}}=-\frac{\beta}{\gamma}$ for $n \geq-k-l$, respectively. Therefore, by taking into account (2.1), we have

$$
v_{n-l}=-\frac{b}{c} \text { and } u_{n-l}=-\frac{\beta}{\gamma}
$$

for $n \in \mathbb{N}_{0}$. Now, we again consider the system (2.2) and the functions

$$
g(t)=\frac{a c t+a b+d}{c t+b}, f(t)=\frac{\alpha \gamma t+\alpha \beta+\delta}{\gamma t+\beta}
$$

which correspond to the equations of (2.2). Hence, we can describe the solutions of (2.7) and (2.8) as follows:

$$
\begin{gathered}
v_{2 m-1}^{(i)}=(f \circ g)^{m}\left(v_{-1}^{(i)}\right), \\
v_{2 m}^{(i)}=\left((f \circ g)^{m} \circ f\right)\left(u_{-1}^{(i)}\right), \\
u_{2 m-1}^{(i)}=(g \circ f)^{m}\left(u_{-1}^{(i)}\right), \\
u_{2 m}^{(i)}=\left((g \circ f)^{m} \circ g\right)\left(v_{-1}^{(i)}\right),
\end{gathered}
$$

for $m \geq 0$. By using (2.29) and the implicit forms (2.30)-(2.33), we have

$$
\begin{gathered}
v_{-1}^{(i)}=(f \circ g)^{-m}\left(-\frac{b}{c}\right), \\
u_{-1}^{(i)}=(g \circ f)^{-m}\left(f^{-1}\left(-\frac{b}{c}\right)\right)=(g \circ f)^{-m}\left(-\frac{b \beta+c \alpha \beta+c \delta}{b \gamma+c \alpha \gamma}\right), \\
u_{-1}^{(i)}=(g \circ f)^{-m}\left(-\frac{\beta}{\gamma}\right), \\
v_{-1}^{(i)}=(f \circ g)^{-m}\left(g^{-1}\left(-\frac{\beta}{\gamma}\right)\right)=(f \circ g)^{-m}\left(-\frac{b \beta+a b \gamma+d \gamma}{c \beta+a c \gamma}\right),
\end{gathered}
$$

where

$$
g^{-1}(t)=\frac{-b t+a b+d}{c t-a c}, f^{-1}(t)=\frac{-\beta t+\alpha \beta+\delta}{\gamma t-\alpha \gamma},
$$

respectively. This means that if one of the conditions in (2.34)-(2.37) holds, then $2 m-t h$ iteration or $(2 m+1)$ - th iteration in $(2.2)$ cannot be calculated. Consequently, desired result follows from (2.1). Also, note that system associated with the functions $f^{-1}$ and $g^{-1}$ is

$$
w_{n}=\frac{-b \widetilde{w}_{n-l}+a b+d}{c \widetilde{w}_{n-l}-a c}, \widetilde{w}_{n}=\frac{-\beta w_{n-l}+\alpha \beta+\delta}{\gamma w_{n-l}-\alpha \gamma}, n \in \mathbb{N}_{0},
$$

and is solvable. That is, the right hand sides of the equalities in (2.34)-(2.37) can be obtained in the closed form.

\section{Some special cases of the system (1.5)}

In this section we deal with some special cases of the system (1.5). We note that the system (1.5) is trivial, if $a=d=0$ or $\alpha=\delta=0$ and can be undefined, if $b=c=0$ or $\beta=\gamma=0$. So, we consider definable cases. 


\subsection{Case $d=0$}

If $d=0$ then the system (1.5) reduces to the following system

$$
x_{n}=a y_{n-k}, y_{n}=\alpha x_{n-k}+\frac{\delta x_{n-k} y_{n-(k+l)}}{\beta y_{n-(k+l)}+\gamma x_{n-l}}, n \in \mathbb{N}_{0} .
$$

By using the changes of variables in (2.1) we get

$$
u_{n}=a, v_{n}=\frac{\alpha \gamma a+\alpha \beta+\delta}{\gamma a+\beta} .
$$

Putting (3.1) in (2.27) and (2.28), we get solutions of the system (1.5).

\subsection{Case $\delta=0$}

If $\delta=0$ then the system (1.5) reduces to the following system

$$
x_{n}=a y_{n-k}+\frac{d y_{n-k} x_{n-(k+l)}}{b x_{n-(k+l)}+c y_{n-l}}, y_{n}=\alpha x_{n-k}, n \in \mathbb{N}_{0} .
$$

This case is dual of previous case. By using the changes of variables in (2.1) we get

$$
u_{n}=\frac{a c \alpha+a b+d}{c \alpha+b}, v_{n}=\alpha
$$

Putting (3.2) in (2.27) and (2.28), we get solutions of the system (1.5).

\subsection{Case $d=\delta=0$}

If $d=\delta=0$ then the system (1.5) reduces to the following linear system

$$
x_{n}=a y_{n-k}, y_{n}=\alpha x_{n-k}, n \in \mathbb{N}_{0},
$$

which is one of simplest cases. By using the changes of variables in (2.1) we get

$$
u_{n}=a, v_{n}=\alpha
$$

Putting (3.3) in (2.27) and (2.28), we get solutions of the system (1.5).

\subsection{Case $c=0$}

If $c=0$ then the system (1.5) reduces to the following system

$$
x_{n}=\left(\frac{a b+d}{b}\right) y_{n-k}, y_{n}=\alpha x_{n-k}+\frac{\delta x_{n-k} y_{n-(k+l)}}{\beta y_{n-(k+l)}+\gamma x_{n-l}}, n \in \mathbb{N}_{0} .
$$

By using the changes of variables in (2.1) we get

$$
u_{n}=\frac{a b+d}{b}, v_{n}=\frac{\alpha \beta b+\alpha \gamma a b+\alpha \gamma d+b \delta}{\beta b+\gamma a b+\gamma d} .
$$

Putting (3.4) in (2.27) and (2.28), we get solutions of the system (1.5).

\subsection{Case $\gamma=0$}

If $\gamma=0$ then the system (1.5) reduces to the following system

$$
x_{n}=a y_{n-k}+\frac{d y_{n-k} x_{n-(k+l)}}{b x_{n-(k+l)}+c y_{n-l}}, y_{n}=\left(\frac{\alpha \beta+\delta}{\beta}\right) x_{n-k}, n \in \mathbb{N}_{0} .
$$

By using the changes of variables in (2.1) we get

$$
u_{n}=\frac{a b \beta+a c \alpha \beta+a c \delta+\beta d}{b \beta+c \alpha \beta+c \delta}, v_{n}=\frac{\alpha \beta+\delta}{\beta} .
$$

Putting (3.5) in (2.27) and (2.28), we get solutions of the system (1.5). 
3.6. Case $c=\gamma=0$

If $c=\gamma=0$ then the system (1.5) reduces to the following system

$$
x_{n}=\left(\frac{a b+d}{b}\right) y_{n-k}, y_{n}=\left(\frac{\alpha \beta+\delta}{\beta}\right) x_{n-k}, n \in \mathbb{N}_{0} .
$$

By using the changes of variables in (2.1) we get

$$
u_{n}=\frac{a b+d}{b}, v_{n}=\frac{\alpha \beta+\delta}{\beta} .
$$

Putting (3.6) in (2.27) and (2.28), we get solutions of the system (1.5).

\subsection{Case $a=c=0$}

If $a=c=0$ then the system (1.5) reduces to the following system

$$
x_{n}=\left(\frac{d}{b}\right) y_{n-k}, y_{n}=\alpha x_{n-k}+\frac{\delta x_{n-k} y_{n-(k+l)}}{\beta y_{n-(k+l)}+\gamma x_{n-l}}, n \in \mathbb{N}_{0} .
$$

By using the changes of variables in (2.1) we get

$$
u_{n}=\frac{d}{b}, v_{n}=\frac{\alpha \gamma d+\alpha \beta b+\delta b}{\gamma d+\beta b} .
$$

Putting (3.7) in (2.27) and (2.28), we get solutions of the system (1.5).

\subsection{Case $\alpha=\gamma=0$}

If $\alpha=\gamma=0$ then the system (1.5) reduces to the following system

$$
x_{n}=a y_{n-k}+\frac{d y_{n-k} x_{n-(k+l)}}{b x_{n-(k+l)}+c y_{n-l}}, y_{n}=\frac{\delta}{\beta} x_{n-k}, n \in \mathbb{N}_{0} .
$$

By using the changes of variables in (2.1) we get

$$
u_{n}=\frac{a c \delta+a b \beta+d \beta}{c \delta+b \beta}, v_{n}=\frac{\delta}{\beta} .
$$

Putting (3.8) in (2.27) and (2.28), we get solutions of the system (1.5).

\subsection{Case $a=c=\alpha=\gamma=0$}

If $a=c=\alpha=\gamma=0$ then the system (1.5) reduces to the following system

$$
x_{n}=\frac{d}{b} y_{n-k}, y_{n}=\frac{\delta}{\beta} x_{n-k}, n \in \mathbb{N}_{0} .
$$

By using the changes of variables in (2.1), we get

$$
u_{n}=\frac{d}{b}, v_{n}=\frac{\delta}{\beta} \text {. }
$$

Putting (3.9) in (2.27) and (2.28), we get solutions of the system (1.5). 
3.10. Case $a=b=0$

If $a=b=0$ then the system (1.5) reduces to the following system

$$
x_{n}=\frac{d y_{n-k} x_{n-(k+l)}}{c y_{n-l}}, y_{n}=\alpha x_{n-k}+\frac{\delta x_{n-k} y_{n-(k+l)}}{\beta y_{n-(k+l)}+\gamma x_{n-l}}, n \in \mathbb{N}_{0} .
$$

By using the changes of variables in (2.1), we get $u_{n}=\frac{d}{c v_{n-l}}$ from the first equation of (3.10) and so

$$
v_{n}=\frac{(\alpha \beta c+\delta c) v_{n-2 l}+\alpha \gamma d}{\beta c v_{n-2 l}+\gamma d}, n \geq l,
$$

from its second equation. Eq. (3.11) can be solved and its solution is given by

$$
v_{2 l m+i}= \begin{cases}\frac{A_{10}}{B_{10}} \frac{P_{101} \lambda_{1}^{m+1}-P_{102} \lambda_{2}^{m+1}}{P_{101} \lambda_{1}^{m}-P_{102} \lambda_{2}^{m}}-\frac{C_{10}}{B_{10}} & \text { if } R_{10} \neq \frac{1}{4}, \\ \frac{A_{10}}{B_{10}}\left(\frac{P_{10}\left(P_{10}-A_{10}\right)(m+1)}{2 P_{10}+\left(2 P_{10}-2 A_{10}\right) m}\right)-\frac{C_{10}}{B_{10}} & \text { if } R_{10}=\frac{1}{4},\end{cases}
$$

where $R_{10}=\frac{\delta c \gamma d}{(\alpha \beta c+\delta c+\gamma d)^{2}}, A_{10}=\alpha \beta c+\delta c+\gamma d, B_{10}=\beta c$ and $C_{10}=\gamma d, P_{10 j}=$ $\lambda_{j} B_{10} \frac{y_{i-2 l}}{x_{i-2 l-k}}+\lambda_{j} C_{10}-R_{10} A_{10}, P_{10}=2 B_{10} \frac{y_{i-2 l}}{x_{i-2 l-k}}+2 C_{10}$ for $j \in\{1,2\}, i=\overline{l, 3 l-1}$. From the equation $u_{n}=\frac{d}{c v_{n-l}}$, we get

$$
u_{2 m+i_{1}}=\frac{d}{c v_{2 m+i_{1}-l}} .
$$

for $2 m \geq-i_{1}$ and so

$$
u_{2 l n+2 r+i_{1}}=\frac{d}{c v_{l(2 n-1)+2 r+i_{1}}} .
$$

for every $n \in \mathbb{N}_{0}, r=\overline{0, l-1}$ and $i_{1} \in\{0,1\}$. From (3.14), we get

$$
u_{2 l n+2 r+i_{1}}= \begin{cases}\frac{d}{c} \frac{1}{\frac{A_{10}}{B_{10}} \frac{\widehat{P}_{101} \lambda_{1}^{n}-\widehat{P}_{102} \lambda_{2}^{n}}{P_{101} \lambda_{1}^{n-1}-\widehat{P}_{102} \lambda_{2}^{n-1}}-\frac{C_{10}}{B_{10}}} & \text { if } R_{10} \neq \frac{1}{4}, \\ \frac{d}{c} \frac{1}{\frac{A_{10}}{B_{10}}\left(\frac{\widehat{P}_{10}+\left(\widehat{P}_{10}-A_{10}\right) n}{2 P_{10}+\left(\widehat{2}_{10}-2 A_{10}\right)(n-1)}\right)-\frac{C_{10}}{B_{10}}} & \text { if } R_{10}=\frac{1}{4} .\end{cases}
$$

where $R_{10}=\frac{\delta c \gamma d}{(\alpha \beta c+\delta c+\gamma d)^{2}}, A_{10}=\alpha \beta c+\delta c+\gamma d, B_{10}=\beta c$ and $C_{10}=\gamma d, \widehat{P}_{10 j}=$ $\lambda_{j} B_{10} \frac{y_{i-2 l}}{x_{i-2 l-k}}+\lambda_{j} C_{10}-R_{10} A_{10}, \widehat{P}_{10}=2 B_{10} \frac{y_{i-2 l}}{x_{i-2 l-k}}+2 C_{10}$ for $r=\overline{0, l-1}, i_{1} \in\{0,1\}$, $j \in\{1,2\}, i=\overline{l, 3 l-1}$. Putting (3.12) and (3.15) in (2.27) and (2.28), we get solutions of the system (1.5).

\subsection{Case $\alpha=\beta=0$}

This case is dual of the previous case and reduces the system (1.5) to the following system

$$
x_{n}=a y_{n-k}+\frac{d y_{n-k} x_{n-(k+l)}}{b x_{n-(k+l)}+c y_{n-l}}, y_{n}=\frac{\delta x_{n-k} y_{n-(k+l)}}{\gamma x_{n-l}}, n \in \mathbb{N}_{0} .
$$

By using the changes of variables in (2.1), the system (3.16) is transformed into the system

$$
u_{n}=\frac{(a b \gamma+d \gamma) u_{n-2 l}+a c \delta}{b \gamma u_{n-2 l}+c \delta}, n \geq l, v_{n}=\frac{\delta}{\gamma u_{n-l}}, n \in \mathbb{N}_{0} .
$$

The first equation of the system (3.17) can be solved and its solution is given by

$$
u_{2 l m+i}= \begin{cases}\frac{A_{11}}{B_{11}} \frac{P_{111} \lambda_{1}^{m+1}-P_{112} \lambda_{2}^{m+1}}{P_{111} \lambda_{1}^{m}-P_{112} \lambda_{2}^{m}}-\frac{C_{11}}{B_{11}} & \text { if } R_{11} \neq \frac{1}{4}, \\ \frac{A_{11}}{B_{11}}\left(\frac{P_{11}+\left(P_{11}-A_{11}\right)(m+1)}{2 P_{11}+\left(2 P_{11}-2 A_{11}\right) m}\right)-\frac{C_{11}}{B_{11}} & \text { if } R_{11}=\frac{1}{4},\end{cases}
$$


where $R_{11}=\frac{d \gamma c \delta}{(a b \gamma+d \gamma+c \delta)^{2}}, A_{11}=a b \gamma+d \gamma+c \delta, B_{11}=b \gamma$ and $C_{11}=c \delta, P_{11 j}=$ $\lambda_{j} B_{11} \frac{x_{i-2 l}}{y_{i-2 l-k}}+\lambda_{j} C_{11}-R_{11} A_{11}, P_{11}=2 B_{11} \frac{x_{i-2 l}}{y_{i-2 l-k}}+2 C_{11}$ for $j \in\{1,2\}, i=\overline{l, 3 l-1}$. From second equation of the system (3.17), we get

$$
v_{2 m+i_{1}}=\frac{\delta}{\gamma u_{2 m+i_{1}-l}},
$$

for $2 m \geq-i_{1}$ and so

$$
v_{2 l n+2 r+i_{1}}=\frac{\delta}{\gamma u_{l(2 n-1)+2 r+i_{1}}},
$$

for every $n \in \mathbb{N}_{0}, r=\overline{0, l-1}$ and $i_{1} \in\{0,1\}$. From (3.20), we get the formulas

$$
v_{2 l n+2 r+i_{1}}= \begin{cases}\frac{\delta}{\gamma} \frac{1}{\frac{A_{11}}{B_{11}} \frac{\widehat{P}_{111} \lambda_{1}^{n}-\widehat{P}_{112} \lambda_{2}^{n}}{\widehat{P}_{111} \lambda_{1}^{n-1}-\widehat{P}_{112} \lambda_{2}^{n-1}-\frac{C_{11}}{B_{11}}}} & \text { if } R_{11} \neq \frac{1}{4}, \\ \frac{\delta}{\gamma} \frac{1}{\frac{A_{11}}{B_{11}}\left(\frac{\widehat{P}_{11}+\left(\widehat{P}_{11}-A_{11}\right) n}{2 \widehat{P}_{11}+\left(2 \widehat{P}_{11}-2 A_{11}\right)(n-1)}\right)-\frac{C_{11}}{B_{11}}} & \text { if } R_{11}=\frac{1}{4},\end{cases}
$$

where $R_{11}=\frac{d \gamma c \delta}{(a b \gamma+d \gamma+c \delta)^{2}}, A_{11}=a b \gamma+d \gamma+c \delta, B_{11}=b \gamma$ and $C_{11}=c \delta, \widehat{P}_{11 j}=$ $\lambda_{j} B_{11} \frac{x_{i-2 l}}{y_{i-2 l-k}}+\lambda_{j} C_{11}-R_{11} A_{11}, \widehat{P}_{11}=2 B_{11} \frac{x_{i-2 l}}{y_{i-2 l-k}}+2 C_{11}$ for $r=\overline{0, l-1}, i_{1} \in\{0,1\}$, $j \in\{1,2\}, i=\overline{l, 3 l-1}$. Putting (3.18) and (3.21) in (2.27) and (2.28), we get solutions of the system (1.5).

\subsection{Case $a=b=\alpha=\beta=0$}

In this case, the system (1.5) reduces to the following system

$$
x_{n}=\frac{d y_{n-k} x_{n-(k+l)}}{c y_{n-l}}, y_{n}=\frac{\delta x_{n-k} y_{n-(k+l)}}{\gamma x_{n-l}}, n \in \mathbb{N}_{0} .
$$

By using the changes of variables in (2.1), we get the $2 l$-order linear difference equations

$$
u_{n}=\frac{d \gamma}{c \delta} u_{n-2 l}, v_{n}=\frac{c \delta}{\gamma d} v_{n-2 l}, n \geq l
$$

after some simple operations. From which it follows that

$$
u_{2 l m+i}=\left(\frac{d \gamma}{c \delta}\right)^{m+1} \frac{x_{i-2 l}}{y_{i-k-2 l}}, v_{2 l m+i}=\left(\frac{c \delta}{\gamma d}\right)^{m+1} \frac{y_{i-2 l}}{x_{i-k-2 l}},
$$

where $m \in \mathbb{N}_{0}$ and $i \in\{l, l+1, \ldots, 3 l-1\}$. Putting (3.23) in (2.27) and (2.28), we get solutions of the system (1.5).

\subsection{Case $b=0$}

If $b=0$, then the system (1.5) reduces to the following system

$$
x_{n}=a y_{n-k}+\frac{d y_{n-k} x_{n-(k+l)}}{c y_{n-l}}, y_{n}=\alpha x_{n-k}+\frac{\delta x_{n-k} y_{n-(k+l)}}{\beta y_{n-(k+l)}+\gamma x_{n-l}}, n \in \mathbb{N}_{0} .
$$

By applying (2.1) to (3.24), we get the system

$$
u_{n}=\frac{a c v_{n-l}+d}{c v_{n-l}}, v_{n}=\frac{\alpha \gamma u_{n-l}+\alpha \beta+\delta}{\gamma u_{n-l}+\beta}, n \in \mathbb{N}_{0} .
$$

From (3.25), we get the equations

$$
\begin{aligned}
& u_{n}=\frac{(a c \alpha \gamma+d \gamma) u_{n-2 l}+a c \alpha \beta+a c \delta+\beta d}{c \alpha \gamma u_{n-2 l}+c \alpha \beta+c \delta}, \\
& v_{n}=\frac{(\alpha \gamma a c+\alpha \beta c+\delta c) v_{n-2 l}+\alpha \gamma d}{(\gamma a c+\beta c) v_{n-2 l}+\gamma d}, n \geq l
\end{aligned}
$$


Since both equations in (3.26) are solvable, we get the formulas

$$
u_{2 l m+i}= \begin{cases}\frac{A_{13}}{B_{13}} \frac{P_{131} \lambda_{1}^{m+1}-P_{132} \lambda_{2}^{m+1}}{P_{131} \lambda_{1}^{m}-P_{132} \lambda_{2}^{m}}-\frac{C_{13}}{B_{13}} & \text { if } R_{13} \neq \frac{1}{4}, \\ \frac{A_{13}}{B_{13}}\left(\frac{P_{13}+\left(P_{13}-A_{13}\right)(m+1)}{2 P_{13}+\left(2 P_{13}-2 A_{13}\right) m}\right)-\frac{C_{13}}{B_{13}} & \text { if } R_{13}=\frac{1}{4},\end{cases}
$$

and

$$
v_{2 l m+i}= \begin{cases}\frac{A_{13}}{\widehat{B}_{13}} \frac{\widehat{P}_{131} \lambda_{1}^{m+1}-\widehat{P}_{132} \lambda_{2}^{m+1}}{\widehat{P}_{131} \lambda_{1}^{m}-\widehat{P}_{132} \lambda_{2}^{m}}-\frac{\widehat{C}_{13}}{\widehat{B}_{13}} & \text { if } R_{13} \neq \frac{1}{4}, \\ \frac{A_{13}}{\widehat{B}_{13}}\left(\frac{\widehat{P}_{13}+\left(\widehat{P}_{13}-A_{13}\right)(m+1)}{2 \widehat{P}_{13}+\left(2 \widehat{P}_{13}-2 A_{13}\right) m}\right)-\frac{\widehat{C}_{13}}{\widehat{B}_{13}} & \text { if } R_{13}=\frac{1}{4},\end{cases}
$$

where $R_{13}=\frac{d \gamma c \delta}{(a c \alpha \gamma+d \gamma+c \alpha \beta+c \delta)^{2}}, A_{13}=a c \alpha \gamma+d \gamma+c \alpha \beta+c \delta, B_{13}=c \alpha \gamma, C_{13}=c \alpha \beta+c \delta$, $\widehat{B}_{13}=\gamma a c+\beta c, \widehat{C}_{13}=\gamma d, P_{13 j}=\lambda_{j} B_{13} \frac{x_{i-2 l}}{y_{i-2 l-k}}+\lambda_{j} C_{13}-R_{13} A_{13}, \widehat{P}_{13 j}=\lambda_{j} \widehat{B}_{13} \frac{y_{i-2 l}}{x_{i-2 l-k}}+$ $\lambda_{j} \widehat{C}_{13}-R_{13} A_{13}, P_{13}=2 B_{13} \frac{x_{i-2 l}}{y_{i-2 l-k}}+2 C_{13}, \widehat{P}_{13}=2 \widehat{B}_{13} \frac{y_{i-2 l}}{x_{i-2 l-k}}+2 \widehat{C}_{13}$ for $j \in\{1,2\}$, $i \in\{l, l+1, \ldots, 3 l-1\}$. Putting (3.27) and (3.28) in (2.27) and (2.28), we get solutions of the system (1.5).

\subsection{Case $\beta=0$}

This case is dual of the previous case. Hence, in this case, the system (1.5) is as follows:

$$
x_{n}=a y_{n-k}+\frac{d y_{n-k} x_{n-(k+l)}}{b x_{n-(k+l)}+c y_{n-l}}, y_{n}=\alpha x_{n-k}+\frac{\delta x_{n-k} y_{n-(k+l)}}{\gamma x_{n-l}}, n \in \mathbb{N}_{0} .
$$

By using (2.1), we get the system

$$
u_{n}=\frac{a c v_{n-l}+a b+d}{c v_{n-l}+b}, v_{n}=\frac{\alpha \gamma u_{n-l}+\delta}{\gamma u_{n-l}}, n \in \mathbb{N}_{0},
$$

from (3.29). Therefore, from the system (3.30), we get

$$
\begin{aligned}
& u_{n}=\frac{(a c \alpha \gamma+a b \gamma+d \gamma) u_{n-2 l}+a c \delta}{(c \alpha \gamma+b \gamma) u_{n-2 l}+c \delta}, \\
& v_{n}=\frac{(a c \alpha \gamma+\delta c) v_{n-2 l}+a b \alpha \gamma+\alpha \gamma d+\delta b}{\gamma a c v_{n-2 l}+\gamma a b+\gamma d}, n \geq l .
\end{aligned}
$$

Since both equations of (3.31) are solvable, we get the formulas

$$
\begin{aligned}
& u_{2 l m+i}= \begin{cases}\frac{A_{14}}{B_{14}} \frac{P_{141} \lambda_{1}^{m+1}-P_{142} \lambda_{2}^{m+1}}{P_{141} \lambda_{1}^{m}-P_{142} \lambda_{2}^{m}}-\frac{C_{14}}{B_{14}} & \text { if } R_{14} \neq \frac{1}{4}, \\
\frac{A_{14}}{B_{14}}\left(\frac{P_{14}+\left(P_{14}-A_{14}\right)(m+1)}{2 P_{14}+\left(2 P_{14}-2 A_{14}\right) m}\right)-\frac{C_{14}}{B_{14}} & \text { if } R_{14}=\frac{1}{4}\end{cases} \\
& v_{2 l m+i}= \begin{cases}\frac{A_{14}}{\widehat{B}_{14}} \frac{\widehat{P}_{141} \lambda_{1}^{m+1}-\widehat{P}_{142} \lambda_{2}^{m+1}}{\widehat{P}_{141} \lambda_{1}^{m}-\widehat{P}_{142} \lambda_{2}^{m}}-\frac{\widehat{C}_{14}}{\widehat{B}_{14}} & \text { if } R_{14} \neq \frac{1}{4}, \\
\frac{A_{14}}{\widehat{B}_{14}}\left(\frac{\widehat{P}_{14}+\left(\widehat{P}_{14}-A_{14}\right)(m+1)}{2 \widehat{P}_{14}+\left(2 \widehat{P}_{14}-2 A_{14}\right) m}\right)-\frac{\widehat{C}_{14}}{\widehat{B}_{14}} & \text { if } R_{14}=\frac{1}{4},\end{cases}
\end{aligned}
$$

where $R_{14}=\frac{d \gamma c \delta}{(a c \alpha \gamma+a b \gamma+d \gamma+c \delta)^{2}}, A_{14}=a c \alpha \gamma+a b \gamma+d \gamma+c \delta, B_{14}=c \alpha \gamma+b \gamma, C_{14}=c \delta$, $\widehat{B}_{14}=\gamma a c, \widehat{C}_{14}=\gamma a b+\gamma d, P_{14 j}=\lambda_{j} B_{14} \frac{x_{i-2 l}}{y_{i-2 l-k}}+\lambda_{j} C_{14}-R_{14} A_{14}, \widehat{P}_{14 j}=\lambda_{j} \widehat{B}_{14} \frac{y_{i-2 l}}{x_{i-2 l-k}}+$ $\lambda_{j} \widehat{C}_{14}-R_{14} A_{14}, P_{14}=2 B_{14} \frac{x_{i-2 l}}{y_{i-2 l-k}}+2 C_{14}, \widehat{P}_{14}=2 \widehat{B}_{14} \frac{y_{i-2 l}}{x_{i-2 l-k}}+2 \widehat{C}_{14}$ for $j \in\{1,2\}$, $i \in\{l, l+1, \ldots, 3 l-1\}$. Putting (3.32) and (3.33) in (2.27) and (2.28), we get solutions of the system (1.5). 
3.15. Case $b=\beta=0$

If $b=\beta=0$ then the system (1.5) reduces to the following system

$$
x_{n}=a y_{n-k}+\frac{d y_{n-k} x_{n-(k+l)}}{c y_{n-l}}, y_{n}=\alpha x_{n-k}+\frac{\delta x_{n-k} y_{n-(k+l)}}{\gamma x_{n-l}}, n \in \mathbb{N}_{0} .
$$

By using the changes of variables in (2.1) we get

$$
u_{n}=\frac{a c v_{n-l}+d}{c v_{n-l}}, v_{n}=\frac{\alpha \gamma u_{n-l}+\delta}{\gamma u_{n-l}}, n \in \mathbb{N}_{0} .
$$

From the system (3.34) we get

$$
u_{n}=\frac{(a c \alpha \gamma+\gamma d) u_{n-2 l}+a c \delta}{c \alpha \gamma u_{n-2 l}+c \delta}, v_{n}=\frac{(\alpha \gamma a c+\delta c) v_{n-2 l}+\alpha \gamma d}{\gamma a c v_{n-2 l}+\gamma d}, n \geq l .
$$

From (3.35) we get

$$
\begin{gathered}
u_{2 l m+i}= \begin{cases}\frac{A_{15}}{B_{15}} \frac{P_{15} \lambda_{1}^{m+1}-\left(P_{152} \lambda_{2}^{m+1}\right.}{P_{151} \lambda_{1}^{m}-P_{152} \lambda_{2}^{m}}-\frac{C_{15}}{B_{15}} & \text { if } R_{15} \neq \frac{1}{4}, \\
\frac{A_{15}}{B_{15}}\left(\frac{P_{15}+\left(P_{15}-A_{15}\right)(m+1)}{2 P_{15}+\left(2 P_{15}-2 A_{15}\right) m}\right)-\frac{C_{15}}{B_{15}} & \text { if } R_{15}=\frac{1}{4}\end{cases} \\
v_{2 l m+i}= \begin{cases}\frac{A_{15}}{\widehat{B}_{15}} \frac{\widehat{P}_{151} \lambda_{1}^{m+1}-\widehat{P}_{152} \lambda_{2}^{m+1}}{\widehat{P}_{151} \lambda_{1}^{m}-\widehat{P}_{151} \lambda_{2}^{m}}-\frac{\widehat{C}_{15}}{\widehat{B}_{15}} & \text { if } R_{15} \neq \frac{1}{4}, \\
A_{15}\left(\frac{\widehat{P}_{15}+\left(\widehat{P}_{15}-A_{15}\right)(m+1)}{2 \widehat{P}_{15}+\left(2 \widehat{P}_{15}-2 A_{15}\right) m}\right)-\frac{\widehat{C}_{15}}{\widehat{B}_{15}} & \text { if } R_{15}=\frac{1}{4}\end{cases}
\end{gathered}
$$

where $R_{15}=\frac{c d \gamma \delta}{(a c \alpha \gamma+d \gamma+c \delta)^{2}}, A_{15}=a c \alpha \gamma+\gamma d+c \delta, B_{15}=c \alpha \gamma, C_{15}=c \delta, \widehat{B}_{15}=\gamma a c$, $\widehat{C}_{15}=\gamma d, P_{15 j}=\lambda_{j} B_{15} \frac{x_{i-2 l}}{y_{i-2 l-k}}+\lambda_{j} C_{15}-R_{15} A_{15}, \widehat{P}_{15 j}=\lambda_{j} \widehat{B}_{15} \frac{y_{i-2 l}}{x_{i-2 l-k}}+\lambda_{j} \widehat{C}_{15}-R_{15} A_{15}$, $P_{15}=2 B_{15} \frac{x_{i-2 l}}{y_{i-2 l-k}}+2 C_{15}, \widehat{P}_{15}=2 \widehat{B}_{15} \frac{y_{i-2 l}}{x_{i-2 l-k}}+2 \widehat{C}_{15}$ for $j \in\{1,2\}, i \in\{l, l+1, \ldots, 3 l-1\}$. Putting (3.36) and (3.37) in (2.27) and (2.28), we get solutions of the system (1.5).

\subsection{Case $a b c d \neq 0$}

Here we deal with the case when $a b c d \neq 0$. Since in this case the system (1.5) can be written in the form of

$$
x_{n}=a y_{n-k}+\frac{y_{n-k} x_{n-(k+l)}}{b_{1} x_{n-(k+l)}+c_{1} y_{n-l}}, y_{n}=\alpha x_{n-k}+\frac{\delta x_{n-k} y_{n-(k+l)}}{\beta y_{n-(k+l)}+\gamma x_{n-l}}, n \in \mathbb{N}_{0},
$$

with $b_{1}=\frac{b}{d}$ and $c_{1}=\frac{c}{d}$, we may assume that $d=1$. Hence we will consider the system

$$
\begin{aligned}
& x_{n}=a y_{n-k}+\frac{y_{n-k} x_{n-(k+l)}}{b x_{n-(k+l)}+c y_{n-l}}, \\
& y_{n}=\alpha x_{n-k}+\frac{\delta x_{n-k} y_{n-(k+l)}}{\beta y_{n-(k+l)}+\gamma x_{n-l}}, n \in \mathbb{N}_{0},
\end{aligned}
$$

from now on. Moreover, the system (3.38) can be written in the form of

$$
\begin{aligned}
\frac{x_{n}}{y_{n-k}} & =\frac{(a b \gamma+\gamma+a c \alpha \gamma) \frac{x_{n-2 l}}{y_{n-(k+2 l)}}+a b \beta+\beta+a c \alpha \beta+a c \delta}{(b \gamma+c \alpha \gamma) \frac{x_{n-2 l}}{y_{n-(k+2 l)}}+b \beta+c \alpha \beta+c \delta}, \\
\frac{y_{n}}{x_{n-k}} & =\frac{\alpha \gamma \frac{x_{n-l}}{y_{n-(k+l)}}+(\alpha \beta+\delta)}{\gamma \frac{x_{n-l}}{y_{n-(k+l)}}+\beta}, n \geq l .
\end{aligned}
$$


Remark 3.1. For $a b c \alpha \beta \gamma \delta \neq 0$ in the system (1.5), it is easy to see that there is the degenerate case

$$
\left|\begin{array}{cc}
a c \alpha \gamma+a b \gamma+d \gamma & a c \alpha \beta+a c \delta+a b \beta+d \beta \\
c \alpha \gamma+b \gamma & c \alpha \beta+c \delta+b \beta
\end{array}\right|=0
$$

if and only if $d=0$. Hence, we avoid the degenerate case via the assumption $d \neq 0$.

3.16.1. The case $a b \gamma+\gamma+a c \alpha \gamma+b \beta+c \alpha \beta+c \delta=0$. If $a b \gamma+\gamma+a c \alpha \gamma+b \beta+c \alpha \beta+c \delta=0$, then we get the system

$$
\begin{aligned}
\frac{x_{n}}{y_{n-k}} & =\frac{-(b \beta+c \alpha \beta+c \delta) \frac{x_{n-2 l}}{y_{n-(k+2 l)}}+\frac{-\beta(b \beta+c \alpha \beta+c \delta)+a c \gamma \delta}{\gamma}}{(b \gamma+c \alpha \gamma) \frac{x_{n-2 l}}{y_{n-(k+2 l)}}+b \beta+c \alpha \beta+c \delta}, \\
\frac{y_{n}}{x_{n-k}} & =\frac{\alpha \gamma \frac{x_{n-l}}{y_{n-(k+l)}}+(\alpha \beta+\delta)}{\gamma \frac{x_{n-l}}{y_{n-(k+l)}}+\beta}, n \geq l,
\end{aligned}
$$

from (3.39). By using the change of variables (2.1), we get the system

$$
\begin{aligned}
& u_{n}=\frac{-(b \beta+c \alpha \beta+c \delta) u_{n-2 l}+\frac{-\beta(b \beta+c \alpha \beta+c \delta)+a c \gamma \delta}{\gamma}}{(b \gamma+c \alpha \gamma) u_{n-2 l}+b \beta+c \alpha \beta+c \delta}, \\
& v_{n}=\frac{\alpha \gamma u_{n-l}+(\alpha \beta+\delta)}{\gamma u_{n-l}+\beta}, n \geq l
\end{aligned}
$$

which can be written as

$$
\begin{gathered}
(b \gamma+c \alpha \gamma) u_{n}+b \beta+c \alpha \beta+c \delta=\frac{a b c \gamma \delta+a c^{2} \alpha \gamma \delta+c^{2} \alpha \beta \delta+c \delta b \beta+c^{2} \delta^{2}}{(b \gamma+c \alpha \gamma) u_{n-2 l}+b \beta+c \alpha \beta+c \delta}, \\
v_{n}=\frac{\alpha \gamma u_{n-l}+(\alpha \beta+\delta)}{\gamma u_{n-l}+\beta}, n \geq l .
\end{gathered}
$$

By applying the change of variables $(b \gamma+c \alpha \gamma) u_{n}+b \beta+c \alpha \beta+c \delta=t_{n}$ to the system (3.42), we obtain

$$
\begin{aligned}
t_{n} & =\frac{a b c \gamma \delta+a c^{2} \alpha \gamma \delta+c^{2} \alpha \beta \delta+c \delta b \beta+c^{2} \delta^{2}}{t_{n-2 l}} \\
& =t_{n-4 l} \\
& =c_{j}, n \geq 3 l, j=\overline{1,4 l},
\end{aligned}
$$

where each $c_{j}$ is a constant which dependents to the initial values $x_{-i}, y_{-i}, i=\overline{1, k+l}$. Consequently, by using $(b \gamma+c \alpha \gamma) u_{n}+b \beta+c \alpha \beta+c \delta=t_{n}$ and considering the system (3.42), we get

$$
u_{n}=\frac{t_{n}-(b \beta+c \alpha \beta+c \delta)}{b \gamma+c \alpha \gamma}, n \geq-l, v_{n}=\frac{\alpha t_{n-l}+\delta b}{t_{n-l}-c \delta}, n \in \mathbb{N}_{0}
$$

Putting (3.44) in (2.27) and (2.28), we get solutions of the system (1.5).

\subsection{Case $\alpha \beta \gamma \delta \neq 0$}

Here we deal with the case when $\alpha \beta \gamma \delta \neq 0$. Since in this case the system (1.5) can be written in the form of

$$
x_{n}=a y_{n-k}+\frac{d y_{n-k} x_{n-(k+l)}}{b x_{n-(k+l)}+c y_{n-l}}, y_{n}=\alpha x_{n-k}+\frac{x_{n-k} y_{n-(k+l)}}{\beta_{1} y_{n-(k+l)}+\gamma_{1} x_{n-l}}, n \in \mathbb{N}_{0},
$$


with $\beta_{1}=\frac{\beta}{\delta}$ and $\gamma_{1}=\frac{\gamma}{\delta}$, we may assume that $\delta=1$. Hence we will consider the system

$$
\begin{aligned}
& x_{n}=a y_{n-k}+\frac{d y_{n-k} x_{n-(k+l)}}{b x_{n-(k+l)}+c y_{n-l}}, \\
& y_{n}=\alpha x_{n-k}+\frac{x_{n-k} y_{n-(k+l)}}{\beta y_{n-(k+l)}+\gamma x_{n-l}}, n \in \mathbb{N}_{0},
\end{aligned}
$$

from now on. Moreover, the system (3.45) can be written in the form of

$$
\begin{aligned}
\frac{x_{n}}{y_{n-k}} & =\frac{a c \frac{y_{n-l}}{x_{n-(k+l)}}+(a b+d)}{c \frac{y_{n-l}}{x_{n-(k+l)}}+b}, \\
\frac{y_{n}}{x_{n-k}} & =\frac{(\alpha \beta c+c+\alpha \gamma a c) \frac{y_{n-2 l}}{x_{n-(k+2 l)}}+\alpha \beta b+b+\alpha \gamma a b+\alpha \gamma d}{(\beta c+\gamma a c) \frac{y_{n-2 l}}{x_{n-(k+2 l)}}+\beta b+\gamma a b+\gamma d}, n \geq l .
\end{aligned}
$$

Remark 3.2. For $\alpha \beta \gamma a b c d \neq 0$ in the system (1.5), it is easy to see that there is the degenerate case

$$
\left|\begin{array}{cc}
\alpha \gamma a c+\alpha \beta c+\delta c & \alpha \gamma a b+\alpha \gamma d+\alpha \beta b+\delta b \\
\gamma a c+\beta c & \gamma a b+\gamma d+\beta b
\end{array}\right|=0
$$

if and only if $\delta=0$. Hence, we avoid the degenerate case via the assumption $\delta \neq 0$.

3.17.1. The case $\alpha \beta c+c+\alpha \gamma a c+\beta b+\gamma a b+\gamma d=0$. If $\alpha \beta c+c+\alpha \gamma a c+\beta b+\gamma a b+\gamma d=0$, then we get the system

$$
\begin{aligned}
\frac{x_{n}}{y_{n-k}} & =\frac{a c \frac{y_{n-l}}{x_{n-(k+l)}}+(a b+d)}{c \frac{y_{n-l}}{x_{n-(k+l)}}+b}, \\
\frac{y_{n}}{x_{n-k}} & =\frac{-(\beta b+\gamma a b+\gamma d) \frac{y_{n-2 l}}{x_{n-(k+2 l)}}+\frac{-b(\beta b+\gamma a b+\gamma d)+\alpha \gamma c d}{c}}{(\beta c+\gamma a c) \frac{y_{n-2 l}}{x_{n-(k+2 l)}}+\beta b+\gamma a b+\gamma d}, n \geq l,
\end{aligned}
$$

from (3.46). By using the change of variables (2.1), we get the system

$$
\begin{aligned}
& u_{n}=\frac{a c v_{n-l}+(a b+d)}{c v_{n-l}+b}, \\
& v_{n}=\frac{-(\beta b+\gamma a b+\gamma d) v_{n-2 l}+\frac{-b(\beta b+\gamma a b+\gamma d)+\alpha \gamma c d}{c}}{(\beta c+\gamma a c) v_{n-2 l}+\beta b+\gamma a b+\gamma d}, n \geq l,
\end{aligned}
$$

which can be written as

$$
\begin{gathered}
u_{n}=\frac{a c v_{n-l}+(a b+d)}{c v_{n-l}+b}, n \geq l, \\
(\beta c+\gamma a c) v_{n}+\beta b+\gamma a b+\gamma d=\frac{\alpha \beta \gamma c d+\alpha \gamma^{2} a c d+\gamma^{2} a b d+\gamma d \beta b+\gamma^{2} d^{2}}{(\beta c+\gamma a c) v_{n-2 l}+\beta b+\gamma a b+\gamma d}, n \geq l .
\end{gathered}
$$

Next, by applying the change of variables $(\beta c+\gamma a c) v_{n}+\beta b+\gamma a b+\gamma d=\widehat{t}_{n}$ to the system (3.49), we obtain

$$
\begin{aligned}
\widehat{t}_{n} & =\frac{\alpha \beta \gamma c d+\alpha \gamma^{2} a c d+\gamma^{2} a b d+\gamma d \beta b+\gamma^{2} d^{2}}{\widehat{t}_{n-2 l}} \\
& =\widehat{t}_{n-4 l} \\
& =d_{j}, n \geq 3 l, j=\overline{1,4 l},
\end{aligned}
$$

where each $d_{j}$ is a constant which dependents to the initial values $x_{-i}, y_{-i}, i=\overline{1, k+l}$. Consequently, by using $(\beta c+\gamma a c) v_{n}+\beta b+\gamma a b+\gamma d=\widehat{t}_{n}$ and considering the system 
(3.49), we get

$$
u_{n}=\frac{a \widehat{t}_{n-l}+d \beta}{\widehat{t}_{n-l}-\gamma d}, n \in \mathbb{N}_{0}, v_{n}=\frac{\widehat{t}_{n}-(\beta b+\gamma a b+\gamma d)}{\beta c+\gamma a c}, n \geq-l .
$$

Putting (3.51) in (2.27) and (2.28), we get solutions of the system (1.5).

\subsection{Case $a b c d \alpha \beta \gamma \delta \neq 0$}

Here we deal with the case when $a b c d \alpha \beta \gamma \delta \neq 0$. Since in this case the system (1.5) can be written in the form of

$$
x_{n}=a y_{n-k}+\frac{y_{n-k} x_{n-(k+l)}}{b_{2} x_{n-(k+l)}+c_{2} y_{n-l}}, y_{n}=\alpha x_{n-k}+\frac{x_{n-k} y_{n-(k+l)}}{\beta_{2} y_{n-(k+l)}+\gamma_{2} x_{n-l}}, n \in \mathbb{N}_{0},
$$

with $b_{2}=\frac{b}{d}, c_{2}=\frac{c}{d}, \beta_{2}=\frac{\beta}{\delta}$ and $\gamma_{2}=\frac{\gamma}{\delta}$, we may assume that $d=1$ and $\delta=1$. Hence we will consider the system

$$
\begin{aligned}
& x_{n}=a y_{n-k}+\frac{y_{n-k} x_{n-(k+l)}}{b x_{n-(k+l)}+c y_{n-l}}, \\
& y_{n}=\alpha x_{n-k}+\frac{x_{n-k} y_{n-(k+l)}}{\beta y_{n-(k+l)}+\gamma x_{n-l}}, n \in \mathbb{N}_{0},
\end{aligned}
$$

from now on. Moreover, the system (3.52) can be written in the form of

$$
\begin{gathered}
\frac{x_{n}}{y_{n-k}}=\frac{(a b \gamma+a c \alpha \gamma+\gamma) \frac{x_{n-2 l}}{y_{n-(k+2 l)}}+a b \beta+\beta+a c \alpha \beta+a c}{(b \gamma+c \alpha \gamma) \frac{x_{n-2 l}}{y_{n-(k+2 l)}}+b \beta+c \alpha \beta+c}, n \geq l, \\
\frac{y_{n}}{x_{n-k}}=\frac{(\alpha \beta c+\alpha \gamma a c+c) \frac{y_{n-2 l}}{x_{n-(k+2 l)}}+\alpha \beta b+b+\alpha \gamma a b+\alpha \gamma}{(\beta c+\gamma a c) \frac{y_{n-2 l}}{x_{n-(k+2 l)}}+\beta b+\gamma a b+\gamma}, n \geq l .
\end{gathered}
$$

Remark 3.3. For $a b c \alpha \beta \gamma \neq 0$ in the system (1.5), it is easy to see that there is the degenerate case

$$
\begin{aligned}
& \left|\begin{array}{cc}
a c \alpha \gamma+a b \gamma+d \gamma & a c \alpha \beta+a c \delta+a b \beta+d \beta \\
c \alpha \gamma+b \gamma & c \alpha \beta+c \delta+b \beta
\end{array}\right|=0, \\
& \left|\begin{array}{cc}
\alpha \gamma a c+\alpha \beta c+\delta c & \alpha \gamma a b+\alpha \gamma d+\alpha \beta b+\delta b \\
\gamma a c+\beta c & \gamma a b+\gamma d+\beta b
\end{array}\right|=0,
\end{aligned}
$$

if and only if $d=0$ and $\delta=0$. Hence, we avoid the degenerate case via the assumptions $d \neq 0$ and $\delta \neq 0$.

3.18.1. The case $a b \gamma+a c \alpha \gamma+b \beta+c \alpha \beta+c+\gamma=0$. If $a b \gamma+a c \alpha \gamma+b \beta+c \alpha \beta+c+\gamma=0$, then we get the system

$$
\begin{aligned}
& \frac{x_{n}}{y_{n-k}}= \frac{-(b \beta+c \alpha \beta+c) \frac{x_{n-2 l}}{y_{n-(k+2 l)}}+\frac{-\beta(b \beta+c \alpha \beta+c)+a c \gamma}{\gamma}}{(b \gamma+c \alpha \gamma) \frac{x_{n-2 l}}{y_{n-(k+2 l)}}+b \beta+c \alpha \beta+c}, n \geq l, \\
& \frac{y_{n}}{x_{n-k}}=\frac{-(\beta b+\gamma a b+\gamma) \frac{y_{n-2 l}}{x_{n-(k+2 l)}}+\frac{-b(\beta b+\gamma a b+\gamma)+\alpha \gamma c}{c}}{(\beta c+\gamma a c) \frac{y_{n-2 l}}{x_{n-(k+2 l)}}+\beta b+\gamma a b+\gamma}, n \geq l,
\end{aligned}
$$

from (3.53). By using the change of variables (2.1), we get the system

$$
\begin{aligned}
& u_{n}=\frac{-(b \beta+c \alpha \beta+c) u_{n-2 l}+\frac{-\beta(b \beta+c \alpha \beta+c)+a c \gamma}{\gamma}}{(b \gamma+c \alpha \gamma) u_{n-2 l}+b \beta+c \alpha \beta+c}, n \geq l, \\
& v_{n}=\frac{-(\beta b+\gamma a b+\gamma) v_{n-2 l}+\frac{-b(\beta b+\gamma a b+\gamma)+\alpha \gamma c}{c}}{(\beta c+\gamma a c) v_{n-2 l}+\beta b+\gamma a b+\gamma}, n \geq l,
\end{aligned}
$$


which can be written as

$$
\begin{aligned}
& (b \gamma+c \alpha \gamma) u_{n}+b \beta+c \alpha \beta+c=\frac{a b c \gamma+a c^{2} \alpha \gamma+c b \beta+c^{2} \alpha \beta+c^{2}}{(b \gamma+c \alpha \gamma) u_{n-2 l}+b \beta+c \alpha \beta+c}, n \geq l, \\
& (\beta c+\gamma a c) v_{n}+\beta b+\gamma a b+\gamma=\frac{\alpha \beta \gamma c+\alpha \gamma^{2} a c+\gamma \beta b+\gamma^{2} a b+\gamma^{2}}{(\beta c+\gamma a c) v_{n-2 l}+\beta b+\gamma a b+\gamma}, n \geq l .
\end{aligned}
$$

Next, by applying the change of variables $(b \gamma+c \alpha \gamma) u_{n}+b \beta+c \alpha \beta+c=k_{n}$ and $(\beta c+\gamma a c) v_{n}+$ $\beta b+\gamma a b+\gamma=\widehat{k}_{n}$ to the system (3.56), we obtain

$$
\begin{aligned}
& k_{n}=\frac{a b c \gamma+a c^{2} \alpha \gamma+c b \beta+c^{2} \alpha \beta+c^{2}}{k_{n-2 l}}=k_{n-4 l}=e_{j}, n \geq 3 l, j=\overline{1,4 l}, \\
& \widehat{k}_{n}=\frac{\alpha \beta \gamma c+\alpha \gamma^{2} a c+\gamma \beta b+\gamma^{2} a b+\gamma^{2}}{\widehat{k}_{n-2 l}}=\widehat{k}_{n-4 l}=\widehat{e}_{j}, n \geq 3 l, j=\overline{1,4 l},
\end{aligned}
$$

where each $e_{j}$ and $\widehat{e}_{j}$ are constants which dependent to the initial values $x_{-i}, y_{-i}, i=$ $\overline{1, k+l}$. Consequently, by using $(b \gamma+c \alpha \gamma) u_{n}+b \beta+c \alpha \beta+c=k_{n}$ and $(\beta c+\gamma a c) v_{n}+$ $\beta b+\gamma a b+\gamma=\widehat{k}_{n}$, we get

$$
u_{n}=\frac{k_{n}-(b \beta+c \alpha \beta+c)}{b \gamma+c \alpha \gamma}, v_{n}=\frac{\widehat{k}_{n}-(\beta b+\gamma a b+\gamma)}{\beta c+\gamma a c}, n \geq-l .
$$

Putting (3.58) in (2.27) and (2.28), we get solutions of the system (1.5).

\section{Long-term behavior of the system for $a b c \alpha \beta \gamma \neq 0$ when $l=1$}

In this section, we investigate the long-term behavior of the solutions of the system (1.5) when $l=1, a b c \alpha \beta \gamma \neq 0$. In this case, we get the system

$$
x_{n}=a y_{n-k}+\frac{y_{n-k} x_{n-k-1}}{b x_{n-k-1}+c y_{n-1}}, y_{n}=\alpha x_{n-k}+\frac{x_{n-k} y_{n-k-1}}{\beta y_{n-k-1}+\gamma x_{n-1}}, n \in \mathbb{N}_{0} .
$$

The solutions of the system (4.1) are given by

$$
x_{2 k n+2 r+i}=\left\{\begin{aligned}
x_{2 r-2 k+i} \prod_{j=0}^{n}\left(\frac{D}{E_{1}} \frac{N_{1} \lambda_{1}^{k j+r+1}-N_{2} \lambda_{2}^{k j+r+1}}{N_{1} \lambda_{1}^{k j+r}-N_{2} \lambda_{2}^{k j+r}}-\frac{F_{1}}{E_{1}}\right) & \\
\times\left(\frac{D}{E_{2}} \frac{T_{1} \lambda_{1}^{k j+r+\left\lfloor\frac{-k-1}{2}\right\rfloor+1}-T_{2} \lambda_{2}^{j+r+\left\lfloor\frac{i-k-1}{2}\right\rfloor+1}}{\left.T_{1} \lambda_{1}^{k j+r+\left\lfloor\frac{i-k-1}{2}\right\rfloor}-T_{2} \lambda_{2}^{k j+r+\left\lfloor\frac{i-k-1}{2}\right\rfloor}-\frac{F_{2}}{E_{2}}\right)}\right. & \text { if } \widetilde{R}<\frac{1}{4}, \\
x_{2 r-2 k+i} \prod_{j=0}^{n}\left(\frac{D}{E_{1}}\left(\frac{Z_{1}+\left(Z_{1}-D\right)(k j+r+1)}{2 Z_{1}+\left(2 Z_{1}-2 D\right)(k j+r)}\right)-\frac{F_{1}}{E_{1}}\right) & \\
\times\left(\frac{D}{E_{2}}\left(\frac{Z_{2}+\left(Z_{2}-D\right)\left(k j+r+\left\lfloor\frac{i-k-1}{2}\right\rfloor+1\right)}{2 Z_{2}+\left(2 Z_{2}-2 D\right)\left(k j+r+\left\lfloor\frac{i-k-1}{2}\right\rfloor\right)}\right)-\frac{F_{2}}{E_{2}}\right) & \text { if } \widetilde{R}=\frac{1}{4},
\end{aligned}\right.
$$

and

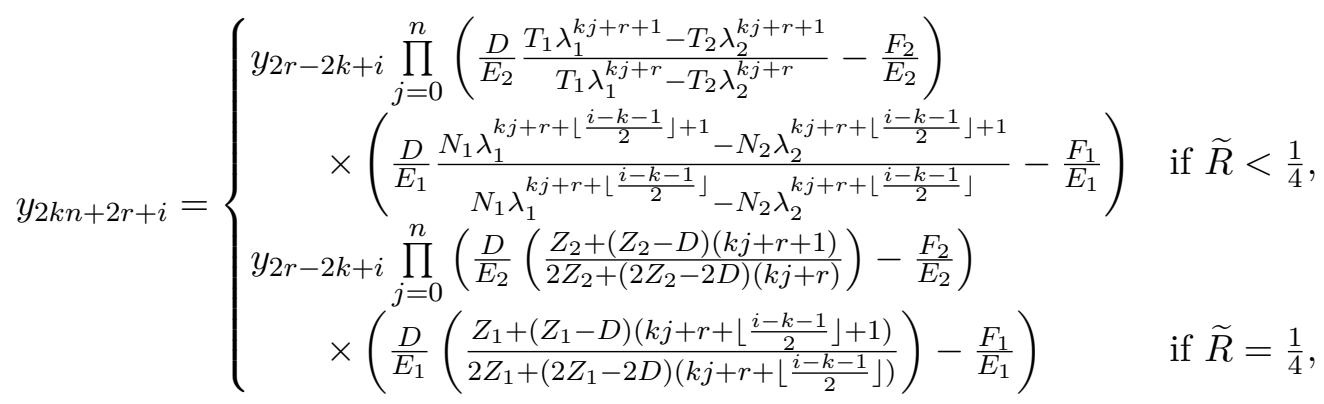

where $\widetilde{R}=\frac{c \gamma}{(a c \alpha \gamma+a b \gamma+\gamma+c \alpha \beta+c+b \beta)^{2}}, D=a c \alpha \gamma+a b \gamma+c \alpha \beta+b \beta+c+\gamma, E_{1}=c \alpha \gamma+b \gamma$, $F_{1}=c \alpha \beta+c+b \beta, E_{2}=\gamma a c+\beta c, F_{2}=\gamma a b+\gamma+\beta b, Z_{1}=2 E_{1} \frac{x_{i-2}}{y_{i-k-2}}+2 F_{1}, Z_{2}=$ $2 E_{2} \frac{y_{i-2}}{x_{i-k-2}}+2 F_{2}, N_{j}:=\lambda_{j} E_{1} \frac{x_{i-2}}{y_{i-k-2}}+\lambda_{j} F_{1}-\widetilde{R} D$ and $T_{j}:=\lambda_{j} E_{2} \frac{y_{i-2}}{x_{i-k-2}}+\lambda_{j} F_{2}-\widetilde{R} D$ for 
$i \in\{1,2\}, j \in\{1,2\}, k \in \mathbb{N}$ and $r \in \mathbb{S}_{k, i}$, where

$\mathbb{S}_{k, i}= \begin{cases}\left\{\frac{k-i-1}{2}+i_{1} ; i_{1}=\overline{0, k-1}\right\} & \text { if }(k-i) \text { odd, } \\ \left\{\left\lfloor\frac{k-i-1}{2}\right\rfloor+i_{1} ; i_{1}=\overline{1, k}\right\} & \text { if }(k-i) \text { even. }\end{cases}$

Theorem 4.1. Assume that $\widetilde{R}=\frac{c \gamma}{(a c \alpha \gamma+a b \gamma+\gamma+c \alpha \beta+c+b \beta)^{2}}<\frac{1}{4}, \frac{x_{i-2}}{y_{i-k-2}} \neq \frac{\widetilde{R} D-\lambda_{j} F_{1}}{\lambda_{j} E_{1}}, \frac{y_{i-2}}{x_{i-k-2}} \neq$ $\frac{\widetilde{R} D-\lambda_{j} F_{2}}{\lambda_{j} E_{2}}, L_{j}:=\frac{D \lambda_{j}-F_{1}}{E_{1}}, M_{j}:=\frac{D \lambda_{j}-F_{2}}{E_{2}}, N_{j}:=\lambda_{j} E_{1} \frac{x_{i-2}}{y_{i-k-2}}+\lambda_{j} F_{1}-\widetilde{R} D$ and $T_{j}:=$ $\lambda_{j} E_{2} \frac{y_{i-2}}{x_{i-k-2}}+\lambda_{j} F_{2}-\widetilde{R} D$ for $i \in\{1,2\}$ and $j \in\{1,2\}$. Then the following statements are true.

(a) If $\left|\lambda_{1}\right|>\left|\lambda_{2}\right|$ and $\left|L_{1} M_{1}\right|<1$, then $x_{n} \rightarrow 0$ and $y_{n} \rightarrow 0$, as $n \rightarrow \infty$.

(b) If $\left|\lambda_{1}\right|>\left|\lambda_{2}\right|$ and $\left|L_{1} M_{1}\right|>1$, then $\left|x_{n}\right| \rightarrow \infty$ and $\left|y_{n}\right| \rightarrow \infty$, as $n \rightarrow \infty$.

(c) If $\left|\lambda_{1}\right|>\left|\lambda_{2}\right|$ and $L_{1} M_{1}=1$, then $\left(x_{n}\right)_{n \geq-k-1}$ and $\left(y_{n}\right)_{n \geq-k-1}$ are convergent.

(d) If $\left|\lambda_{1}\right|>\left|\lambda_{2}\right|$ and $L_{1} M_{1}=-1$, then $\left(x_{2 k n+2 r+i}\right)_{n \in \mathbb{N}_{0}}$ and $\left(y_{2 k n+2 r+i}\right)_{n \in \mathbb{N}_{0}}$, for $i \in\{1,2\}, r \in \mathbb{S}_{k, i}$ are convergent.

(e) If $\left|\lambda_{1}\right|<\left|\lambda_{2}\right|$ and $\left|L_{2} M_{2}\right|<1$, then $x_{n} \rightarrow 0$ and $y_{n} \rightarrow 0$, as $n \rightarrow \infty$.

(f) If $\left|\lambda_{1}\right|<\left|\lambda_{2}\right|$ and $\left|L_{2} M_{2}\right|>1$, then $\left|x_{n}\right| \rightarrow \infty$ and $\left|y_{n}\right| \rightarrow \infty$, as $n \rightarrow \infty$.

(g) If $\left|\lambda_{1}\right|<\left|\lambda_{2}\right|$ and $L_{2} M_{2}=1$, then $\left(x_{n}\right)_{n>-k-1}$ and $\left(y_{n}\right)_{n>-k-1}$ are convergent.

(h) If $\left|\lambda_{1}\right|<\left|\lambda_{2}\right|$ and $L_{2} M_{2}=-1$, then $\left(x_{2 k n+2 r+i}\right)_{n \in \mathbb{N}_{0}}$ and $\left(y_{2 k n+2 r+i}\right)_{n \in \mathbb{N}_{0}}$, for $i \in\{1,2\}, r \in \mathbb{S}_{k, i}$ are convergent.

Proof. Suppose that

$$
\begin{aligned}
a_{n_{1}}^{(r)} & =\left(\frac{D}{E_{1}} \frac{N_{1} \lambda_{1}^{k n_{1}+r+1}-N_{2} \lambda_{2}^{k n_{1}+r+1}}{N_{1} \lambda_{1}^{k n_{1}+r}-N_{2} \lambda_{2}^{k n_{1}+r}}-\frac{F_{1}}{E_{1}}\right) \\
& \times\left(\frac{D}{E_{2}} \frac{T_{1} \lambda_{1}^{k n_{1}+r+\left\lfloor\frac{i-k-1}{2}\right\rfloor+1}-T_{2} \lambda_{2}^{k n_{1}+r+\left\lfloor\frac{i-k-1}{2}\right\rfloor+1}}{T_{1} \lambda_{1}^{k n_{1}+r+\left\lfloor\frac{i-k-1}{2}\right\rfloor}-T_{2} \lambda_{2}^{k n_{1}+r+\left\lfloor\frac{i-k-1}{2}\right\rfloor}}-\frac{F_{2}}{E_{2}}\right)
\end{aligned}
$$

and

$$
\begin{aligned}
b_{n_{1}}^{(r)}= & \left(\frac{D}{E_{2}} \frac{T_{1} \lambda_{1}^{k n_{1}+r+1}-T_{2} \lambda_{2}^{k n_{1}+r+1}}{T_{1} \lambda_{1}^{k n_{1}+r}-T_{2} \lambda_{2}^{k n_{1}+r}}-\frac{F_{2}}{E_{2}}\right) \\
& \times\left(\frac{D}{E_{1}} \frac{N_{1} \lambda_{1}^{k n_{1}+r+\left\lfloor\frac{i-k-1}{2}\right\rfloor+1}-N_{2} \lambda_{2}^{k n_{1}+r+\left\lfloor\frac{i-k-1}{2}\right\rfloor+1}}{\left.N_{1} \lambda_{1}^{k n_{1}+r+\left\lfloor\frac{i-k-1}{2}\right\rfloor}-N_{2} \lambda_{2}^{k n_{1}+r+\left\lfloor\frac{i-k-1}{2}\right\rfloor}-\frac{F_{1}}{E_{1}}\right)}\right.
\end{aligned}
$$

for $n_{1} \in \mathbb{N}_{0}, i \in\{1,2\}$ and $r \in \mathbb{S}_{k, i}$. Then, if $\left|\lambda_{1}\right|>\left|\lambda_{2}\right|$, we get

$$
\lim _{n_{1} \rightarrow \infty} a_{n_{1}}^{(r)}=\lim _{n_{1} \rightarrow \infty} b_{n_{1}}^{(r)}=\left(\frac{D \lambda_{1}-F_{1}}{E_{1}}\right)\left(\frac{D \lambda_{1}-F_{2}}{E_{2}}\right)
$$


for each $i \in\{1,2\}$. From (4.2), (4.3), and (4.6), the results follow from the assumptions in $(a)$ and $(b)$. For each $i \in\{1,2\}$ and sufficiently large $n_{1},(4.4)$ and (4.5) can be written as

$$
\begin{aligned}
a_{n_{1}}^{(r)}= & \left(-\frac{F_{1}}{E_{1}}+\frac{D}{E_{1}} \frac{\lambda_{1}\left(1-\frac{N_{2}}{N_{1}}\left(\frac{\lambda_{2}}{\lambda_{1}}\right)^{k n_{1}+r+1}\right)}{1-\frac{N_{2}}{N_{1}}\left(\frac{\lambda_{2}}{\lambda_{1}}\right)^{k n_{1}+r}}\right) \\
& \times\left(-\frac{F_{2}}{E_{2}}+\frac{D}{E_{2}} \frac{\lambda_{1}\left(1-\frac{T_{2}}{T_{1}}\left(\frac{\lambda_{2}}{\lambda_{1}}\right)^{k n_{1}+r+\left\lfloor\frac{i-k-1}{2}\right\rfloor+1}\right)}{\left.1-\frac{T_{2}}{T_{1}}\left(\frac{\lambda_{2}}{\lambda_{1}}\right)^{k n_{1}+r+\left\lfloor\frac{i-k-1}{2}\right\rfloor}\right)}\right) \\
= & \left(-\frac{F_{1}}{E_{1}}+\frac{D \lambda_{1}}{E_{1}}+\frac{D N_{2}}{E_{1} N_{1}}\left(\lambda_{1}-\lambda_{2}\right)\left(\frac{\lambda_{2}}{\lambda_{1}}\right)^{k n_{1}+r}+\mathcal{O}\left(\frac{\lambda_{2}}{\lambda_{1}}\right)^{2 k n_{1}}\right) \\
\times & \left(-\frac{F_{2}}{E_{2}}+\frac{D \lambda_{1}}{E_{2}}+\frac{D T_{2}}{E_{2} T_{1}}\left(\lambda_{1}-\lambda_{2}\right)\left(\frac{\lambda_{2}}{\lambda_{1}}\right)^{k n_{1}+r+\left\lfloor\frac{i-k-1}{2}\right\rfloor}+\mathcal{O}\left(\frac{\lambda_{2}}{\lambda_{1}}\right)^{2 k n_{1}}\right) \\
= & L_{1} M_{1}+L_{1} \frac{D T_{2}}{E_{2} T_{1}}\left(\lambda_{1}-\lambda_{2}\right)\left(\frac{\lambda_{2}}{\lambda_{1}}\right)^{k n_{1}+r+\left\lfloor\frac{i-k-1}{2}\right\rfloor} \\
& +M_{1} \frac{D N_{2}}{E_{1} N_{1}}\left(\lambda_{1}-\lambda_{2}\right)\left(\frac{\lambda_{2}}{\lambda_{1}}\right)^{k n_{1}+r}+\mathcal{O}\left(\frac{\lambda_{2}}{\lambda_{1}}\right)^{2 k n_{1}}
\end{aligned}
$$

and

$$
\begin{aligned}
b_{n_{1}}^{(r)}= & \left(-\frac{F_{2}}{E_{2}}+\frac{D}{E_{2}} \frac{\lambda_{1}\left(1-\frac{T_{2}}{T_{1}}\left(\frac{\lambda_{2}}{\lambda_{1}}\right)^{k n_{1}+r+1}\right)}{1-\frac{T_{2}}{T_{1}}\left(\frac{\lambda_{2}}{\lambda_{1}}\right)^{k n_{1}+r}}\right) \\
& \times\left(-\frac{F_{1}}{E_{1}}+\frac{D}{E_{1}} \frac{\lambda_{1}\left(1-\frac{N_{2}}{N_{1}}\left(\frac{\lambda_{2}}{\lambda_{1}}\right)^{k n_{1}+r+\left\lfloor\frac{i-k-1}{2}\right\rfloor+1}\right)}{\left.1-\frac{N_{2}}{N_{1}}\left(\frac{\lambda_{2}}{\lambda_{1}}\right)^{k n_{1}+r+\left\lfloor\frac{i-k-1}{2}\right\rfloor}\right)}\right) \\
= & \left(-\frac{F_{2}}{E_{2}}+\frac{D \lambda_{1}}{E_{2}}+\frac{D T_{2}}{E_{2} T_{1}}\left(\lambda_{1}-\lambda_{2}\right)\left(\frac{\lambda_{2}}{\lambda_{1}}\right)^{k n_{1}+r}+\mathcal{O}\left(\frac{\lambda_{2}}{\lambda_{1}}\right)^{2 k n_{1}}\right) \\
\times & \left(-\frac{F_{1}}{E_{1}}+\frac{D \lambda_{1}}{E_{1}}+\frac{D N_{2}}{E_{1} N_{1}}\left(\lambda_{1}-\lambda_{2}\right)\left(\frac{\lambda_{2}}{\lambda_{1}}\right)^{k n_{1}+r+\left\lfloor\frac{i-k-1}{2}\right\rfloor}+\mathcal{O}\left(\frac{\lambda_{2}}{\lambda_{1}}\right)^{2 k n_{1}}\right) \\
= & M_{1} L_{1}+M_{1} \frac{D N_{2}}{E_{1} N_{1}}\left(\lambda_{1}-\lambda_{2}\right)\left(\frac{\lambda_{2}}{\lambda_{1}}\right)^{k n_{1}+r+\left\lfloor\frac{i-k-1}{2}\right\rfloor} \\
& +L_{1} \frac{D T_{2}}{E_{2} T_{1}}\left(\lambda_{1}-\lambda_{2}\right)\left(\frac{\lambda_{2}}{\lambda_{1}}\right)^{k n_{1}+r}+\mathcal{O}\left(\frac{\lambda_{2}}{\lambda_{1}}\right)^{2 k n_{1}} .
\end{aligned}
$$


From (4.2), (4.3), (4.7), and (4.8), the results in (c) can be seen easily. For each $i \in\{1,2\}$ and sufficiently large $n_{1},(4.4)$ and (4.5) can be again written as

$$
\begin{aligned}
a_{n_{1}}^{(r)}= & \left(-\frac{F_{1}}{E_{1}}+\frac{D}{E_{1}} \frac{-\lambda_{1}\left(-1+\frac{N_{2}}{N_{1}}\left(\frac{\lambda_{2}}{\lambda_{1}}\right)^{k n_{1}+r+1}\right)}{1-\frac{N_{2}}{N_{1}}\left(\frac{\lambda_{2}}{\lambda_{1}}\right)^{k n_{1}+r}}\right) \\
& \times\left(-\frac{F_{2}}{E_{2}}+\frac{D}{E_{2}} \frac{-\lambda_{1}\left(-1+\frac{T_{2}}{T_{1}}\left(\frac{\lambda_{2}}{\lambda_{1}}\right)^{k n_{1}+r+\left\lfloor\frac{i-k-1}{2}\right\rfloor+1}\right)}{\left.1-\frac{T_{2}}{T_{1}}\left(\frac{\lambda_{2}}{\lambda_{1}}\right)^{k n_{1}+r+\left\lfloor\frac{i-k-1}{2}\right\rfloor}\right)}\right. \\
= & \left(-\frac{F_{1}}{E_{1}}+\frac{D \lambda_{1}}{E_{1}}\left(1-\frac{N_{2}}{N_{1}} \frac{\left(\lambda_{2}-\lambda_{1}\right)}{\lambda_{1}}\right)\left(\frac{\lambda_{2}}{\lambda_{1}}\right)^{k n_{1}+r}+\mathcal{O}\left(\frac{\lambda_{2}}{\lambda_{1}}\right)^{2 k n_{1}}\right) \\
\times & \left(-\frac{F_{2}}{E_{2}}+\frac{D \lambda_{1}}{E_{2}}\left(1-\frac{T_{2}}{T_{1}} \frac{\left(\lambda_{2}-\lambda_{1}\right)}{\lambda_{1}}\right)\left(\frac{\lambda_{2}}{\lambda_{1}}\right)^{k n_{1}+r+\left\lfloor\frac{i-k-1}{2}\right\rfloor}+\mathcal{O}\left(\frac{\lambda_{2}}{\lambda_{1}}\right)^{2 k n_{1}}\right) \\
= & L_{1} M_{1}\left(1-\frac{N_{2}}{N_{1}} \frac{\left(\lambda_{2}-\lambda_{1}\right)}{\lambda_{1}}\left(\frac{\lambda_{2}}{\lambda_{1}}\right)^{k n_{1}+r}-\frac{T_{2}}{T_{1}} \frac{\left(\lambda_{2}-\lambda_{1}\right)}{\lambda_{1}}\left(\frac{\lambda_{2}}{\lambda_{1}}\right)^{k n_{1}+r+\left\lfloor\frac{i-k-1}{2}\right\rfloor}\right. \\
& \left.+\mathcal{O}\left(\frac{\lambda_{2}}{\lambda_{1}}\right)^{2 k n_{1}}\right)
\end{aligned}
$$

and

$$
\begin{aligned}
b_{n_{1}}^{(r)}= & \left(-\frac{F_{2}}{E_{2}}+\frac{D}{E_{2}} \frac{-\lambda_{1}\left(-1+\frac{T_{2}}{T_{1}}\left(\frac{\lambda_{2}}{\lambda_{1}}\right)^{k n_{1}+r+1}\right)}{1-\frac{T_{2}}{T_{1}}\left(\frac{\lambda_{2}}{\lambda_{1}}\right)^{k n_{1}+r}}\right) \\
\times & \left(-\frac{F_{1}}{E_{1}}+\frac{D}{E_{1}} \frac{-\lambda_{1}\left(-1+\frac{N_{2}}{N_{1}}\left(\frac{\lambda_{2}}{\lambda_{1}}\right)^{k n_{1}+r+\left\lfloor\frac{i-k-1}{2}\right\rfloor+1}\right)}{\left.1-\frac{N_{2}}{N_{1}}\left(\frac{\lambda_{2}}{\lambda_{1}}\right)^{k n_{1}+r+\left\lfloor\frac{i-k-1}{2}\right\rfloor}\right)}\right. \\
= & \left(-\frac{F_{2}}{E_{2}}+\frac{D \lambda_{1}}{E_{2}}\left(1-\frac{T_{2}}{T_{1}} \frac{\left(\lambda_{2}-\lambda_{1}\right)}{\lambda_{1}}\right)\left(\frac{\lambda_{2}}{\lambda_{1}}\right)^{k n_{1}+r}+\mathcal{O}\left(\frac{\lambda_{2}}{\lambda_{1}}\right)^{2 k n_{1}}\right) \\
\times & \left(-\frac{F_{1}}{E_{1}}+\frac{D \lambda_{1}}{E_{1}}\left(1-\frac{N_{2}}{N_{1}} \frac{\left(\lambda_{2}-\lambda_{1}\right)}{\lambda_{1}}\right)\left(\frac{\lambda_{2}}{\lambda_{1}}\right)^{k n_{1}+r+\left\lfloor\frac{i-k-1}{2}\right\rfloor}+\mathcal{O}\left(\frac{\lambda_{2}}{\lambda_{1}}\right)^{2 k n_{1}}\right) \\
= & M_{1} L_{1}\left(1-\frac{T_{2}}{T_{1}} \frac{\left(\lambda_{2}-\lambda_{1}\right)}{\lambda_{1}}\left(\frac{\lambda_{2}}{\lambda_{1}}\right)^{k n_{1}+r}-\frac{N_{2}}{N_{1}} \frac{\left(\lambda_{2}-\lambda_{1}\right)}{\lambda_{1}}\left(\frac{\lambda_{2}}{\lambda_{1}}\right)^{k n_{1}+r+\left\lfloor\frac{i-k-1}{2}\right\rfloor}\right. \\
& \left.+\mathcal{O}\left(\frac{\lambda_{2}}{\lambda_{1}}\right)^{2 k n_{1}}\right)
\end{aligned}
$$

From (4.2), (4.3), (4.9), and (4.10), the results in $(d)$ can be seen easily. Proofs of the $(e)-(h)$ are not given in here since they could be obtained similar with proofs of the $(a)$ $(d)$.

Theorem 4.2. Assume that $\widetilde{R}=\frac{c \gamma}{(a c \alpha \gamma+a b \gamma+\gamma+c \alpha \beta+c+b \beta)^{2}}=\frac{1}{4}, x_{i-k-2} y_{i-k-2} \neq 0$ for $i \in\{1,2\}, D=a c \alpha \gamma+a b \gamma+c \alpha \beta+b \beta+c+\gamma, Z_{1}=2 E_{1} \frac{x_{i-2}}{y_{i-k-2}}+2 F_{1}, Z_{2}=2 E_{2} \frac{y_{i-2}}{x_{i-k-2}}+2 F_{2}$. Then the following statements are true.

(a) If $\left|\frac{\left(D-2 F_{1}\right)\left(D-2 F_{2}\right)}{4 E_{1} E_{2}}\right|<1$, then $x_{n} \rightarrow 0$ and $y_{n} \rightarrow 0$, as $n \rightarrow \infty$. 
(b) If $\left|\frac{\left(D-2 F_{1}\right)\left(D-2 F_{2}\right)}{4 E_{1} E_{2}}\right|>1$, then $\left|x_{n}\right| \rightarrow \infty$ and $\left|y_{n}\right| \rightarrow \infty$, as $n \rightarrow \infty$.

(c) If $\left|\frac{\left(D-2 F_{1}\right)\left(D-2 F_{2}\right)}{4 E_{1} E_{2}}\right|=1$ and $\frac{\left(D-2 F_{1}\right)\left(D-2 F_{2}\right)}{2 D\left(D-F_{1}-F_{2}\right)}>0$, then $\left|x_{n}\right| \rightarrow \infty$ and $\left|y_{n}\right| \rightarrow \infty$, as $n \rightarrow \infty$.

(d) If $\left|\frac{\left(D-2 F_{1}\right)\left(D-2 F_{2}\right)}{4 E_{1} E_{2}}\right|=1$ and $\frac{\left(D-2 F_{1}\right)\left(D-2 F_{2}\right)}{2 D\left(D-F_{1}-F_{2}\right)}<0$, then $x_{n} \rightarrow 0$ and $y_{n} \rightarrow 0$, as $n \rightarrow \infty$.

Proof. If $R=\frac{c \gamma}{(a c \alpha \gamma+a b \gamma+\gamma+c \alpha \beta+c+b \beta)^{2}}=\frac{1}{4}$, then we get $\lambda_{1}=\lambda_{2}=\frac{1}{2}$. Let

and

$$
\begin{aligned}
c_{n_{1}}^{(r)}= & \left(\frac{D}{E_{1}} \frac{Z_{1}+\left(Z_{1}-D\right)\left(k n_{1}+r+1\right)}{2 Z_{1}+\left(2 Z_{1}-2 D\right)\left(k n_{1}+r\right)}-\frac{F_{1}}{E_{1}}\right) \\
& \times\left(\frac{D}{E_{2}} \frac{Z_{2}+\left(Z_{2}-D\right)\left(k n_{1}+r+\left\lfloor\frac{i-k-1}{2}\right\rfloor+1\right)}{2 Z_{2}+\left(2 Z_{2}-2 D\right)\left(k n_{1}+r+\left\lfloor\frac{i-k-1}{2}\right\rfloor\right)}-\frac{F_{2}}{E_{2}}\right)
\end{aligned}
$$

$$
\begin{aligned}
d_{n_{1}}^{(r)}= & \left(\frac{D}{E_{2}} \frac{Z_{2}+\left(Z_{2}-D\right)\left(k n_{1}+r++1\right)}{2 Z_{2}+\left(2 Z_{2}-2 D\right)\left(k n_{1}+r\right)}-\frac{F_{2}}{E_{2}}\right) \\
& \times\left(\frac{D}{E_{1}} \frac{Z_{1}+\left(Z_{1}-D\right)\left(k n_{1}+r+\left\lfloor\frac{i-k-1}{2}\right\rfloor+1\right)}{2 Z_{1}+\left(2 Z_{1}-2 D\right)\left(k n_{1}+r+\left\lfloor\frac{i-k-1}{2}\right\rfloor\right)}-\frac{F_{1}}{E_{1}}\right)
\end{aligned}
$$

for every $n_{1} \in \mathbb{N}_{0}, i \in\{1,2\}$ and $r \in \mathbb{S}_{k, i}$. If at least one of the coefficients of $n_{1}$ is different from 0 , then we have

$$
\lim _{n_{1} \rightarrow \infty} c_{n_{1}}^{(r)}=\frac{\left(D-2 F_{1}\right)\left(D-2 F_{2}\right)}{4 E_{1} E_{2}}=\lim _{n_{1} \rightarrow \infty} d_{n_{1}}^{(r)}
$$

for each $i \in\{1,2\}$. Otherwise, when $\frac{x_{i-2}}{y_{i-k-2}}=\frac{D-2 F_{1}}{2 E_{1}}$ and $\frac{y_{i-2}}{x_{i-k-2}}=\frac{D-2 F_{2}}{2 E_{2}}$ for $i \in\{1,2\}$, we get the equality (4.13). From (4.13), the results follow from the assumptions in $(a)$ and $(b)$. Now we consider the other cases. For each $i \in\{1,2\}$ and sufficiently large $n_{1}$, we obtain

$$
\begin{aligned}
c_{n_{1}}^{(r)}=d_{n_{1}}^{(r)} & =\left(-\frac{F_{1}}{E_{1}}+\frac{D}{E_{1}}\left(\frac{1}{2}+\frac{1}{2 k n_{1}}+\mathcal{O}\left(\frac{1}{n_{1}^{2}}\right)\right)\right) \\
& \times\left(-\frac{F_{2}}{E_{2}}+\frac{D}{E_{2}}\left(\frac{1}{2}+\frac{1}{2 k n_{1}}+\mathcal{O}\left(\frac{1}{n_{1}^{2}}\right)\right)\right) \\
& =\left(\frac{D-2 F_{1}}{2 E_{1}}+\frac{D}{2 E_{1} k n_{1}}+\mathcal{O}\left(\frac{1}{n_{1}^{2}}\right)\right)\left(\frac{D-2 F_{2}}{2 E_{2}}+\frac{D}{2 E_{2} k n_{1}}+\mathcal{O}\left(\frac{1}{n_{1}^{2}}\right)\right) \\
& =\frac{\left(D-2 F_{1}\right)\left(D-2 F_{2}\right)}{4 E_{1} E_{2}}\left(1+\frac{\frac{2 D\left(D-F_{1}-F_{2}\right)}{\left(D-2 F_{1}\right)\left(D-2 F_{2}\right)}}{k n_{1}}+\mathcal{O}\left(\frac{1}{n_{1}^{2}}\right)\right) \\
& = \pm\left(1+\frac{1}{\frac{\left(D-2 F_{1}\right)\left(D_{1}-2 F_{2}\right)}{2 D\left(D-F_{1}-F_{2}\right)} k n_{1}}+\mathcal{O}\left(\frac{1}{n_{1}^{2}}\right)\right) \\
& = \pm \exp \left(\frac{1}{\frac{\left(D-2 F_{1}\right)\left(D-2 F_{2}\right)}{2 D\left(D-F_{1}-F_{2}\right)} k n_{1}}+\mathcal{O}\left(\frac{1}{n_{1}^{2}}\right)\right) .
\end{aligned}
$$

From (4.14), by using the fact that $\sum_{j_{1}=1}^{n_{1}}\left(1 / j_{1}\right) \rightarrow \infty$ as $n_{1} \rightarrow \infty$, then the statements easily follow.

\section{Some other applications}

Now, we will give theoretical explanations for the formulas of solutions of difference equations systems given in [41] as some applications of the main results in Section 2. First, we consider $k=l=1, a=\alpha=0$. In this case the system (1.5) becomes

$$
x_{n}=\frac{d y_{n-1} x_{n-2}}{b x_{n-2}+c y_{n-1}}, y_{n}=\frac{\delta x_{n-1} y_{n-2}}{\beta y_{n-2}+\gamma x_{n-1}}, n \in \mathbb{N}_{0} .
$$


5.1. Case $b=c=d=\beta=\gamma=\delta=1$

We will derive the solution forms of the system (5.1) with $b=c=d=\beta=\gamma=\delta=1$, that is, the system

$$
x_{n}=\frac{y_{n-1} x_{n-2}}{x_{n-2}+y_{n-1}}, y_{n}=\frac{x_{n-1} y_{n-2}}{y_{n-2}+x_{n-1}}, n \in \mathbb{N}_{0},
$$

given in [41], through analytical approach. Also, the general solutions of the system (5.2) are expressed in terms of Fibonacci numbers. Now, to begin with, taking $b=c=d=\beta=$ $\gamma=\delta=1$ in (2.12)-(2.13), we have

$$
q_{m+1}-q_{m}+\frac{1}{9} q_{m-1}=0, m \in \mathbb{N}_{0} .
$$

The characteristic equation of (5.3) can be clearly obtained as of the form $\lambda^{2}-\lambda+\frac{1}{9}=0$, with the roots $\lambda_{1}=\frac{3+\sqrt{5}}{6}=\frac{1}{3}\left(\frac{1+\sqrt{5}}{2}\right)^{2}=\frac{\widehat{\alpha}^{2}}{3}$ and $\lambda_{2}=\frac{3-\sqrt{5}}{6}=\frac{1}{3}\left(\frac{1-\sqrt{5}}{2}\right)^{2}=\frac{\widehat{\beta}^{2}}{3}$. On the other hand, taking into account $A=3, B_{1}=B_{2}=1, C_{1}=C_{2}=2$ and the Binet Formula for Fibonacci numbers, we can rewrite the equations in (2.18)-(2.19) as

$$
\begin{aligned}
& u_{2 m+i}=3 \frac{\left(\lambda_{1} u_{i-2}+2 \lambda_{1}-\frac{1}{3}\right) \lambda_{1}^{m+1}-\left(\lambda_{2} u_{i-2}+2 \lambda_{2}-\frac{1}{3}\right) \lambda_{2}^{m+1}}{\left(\lambda_{1} u_{i-2}+2 \lambda_{1}-\frac{1}{3}\right) \lambda_{1}^{m}-\left(\lambda_{2} u_{i-2}+2 \lambda_{2}-\frac{1}{3}\right) \lambda_{2}^{m}}-2, \\
& v_{2 m+i}=3 \frac{\left(\lambda_{1} v_{i-2}+2 \lambda_{1}-\frac{1}{3}\right) \lambda_{1}^{m+1}-\left(\lambda_{2} v_{i-2}+2 \lambda_{2}-\frac{1}{3}\right) \lambda_{2}^{m+1}}{\left(\lambda_{1} v_{i-2}+2 \lambda_{1}-\frac{1}{3}\right) \lambda_{1}^{m}-\left(\lambda_{2} v_{i-2}+2 \lambda_{2}-\frac{1}{3}\right) \lambda_{2}^{m}}-2,
\end{aligned}
$$

for $m \in \mathbb{N}_{0}, i \in\{1,2\}$.

Using the relations $\widehat{\alpha} \widehat{\beta}=-1, \widehat{\alpha}^{2}+\widehat{\beta}=2, \widehat{\beta}^{2}+\widehat{\alpha}=2$ in (5.4)-(5.5) we get

$$
\begin{aligned}
u_{2 m+i} & =\frac{\left(\widehat{\alpha}^{2 m+4}-\widehat{\beta}^{2 m+4}\right) u_{i-2}+\left(\widehat{\alpha}^{2 m+6}-\widehat{\beta}^{2 m+6}\right)-\left(\widehat{\alpha}^{2 m+3}-\widehat{\beta}^{2 m+3}\right)-\left(\widehat{\alpha}^{2 m+2}-\widehat{\beta}^{2 m+2}\right)}{\left(\widehat{\alpha}^{2 m+2}-\widehat{\beta}^{2 m+2}\right) u_{i-2}+\left(\widehat{\alpha}^{2 m+4}-\widehat{\beta}^{2 m+4}\right)-\left(\widehat{\alpha}^{2 m+1}-\widehat{\beta}^{2 m+1}\right)-\left(\widehat{\alpha}^{2 m}-\widehat{\beta}^{2 m}\right)}-2 \\
& =\frac{F_{2 m+4} u_{i-2}+F_{2 m+6}-F_{2 m+3}-F_{2 m+2}}{F_{2 m+2} u_{i-2}+F_{2 m+4}-F_{2 m+1}-F_{2 m}}-2 \\
& =\frac{F_{2 m+1} u_{i-2}+F_{2 m+2}}{F_{2 m+2} u_{i-2}+F_{2 m+3}}, \\
v_{2 m+i} & =\frac{\left(\widehat{\alpha}^{2 m+4}-\widehat{\beta}^{2 m+4}\right) v_{i-2}+\left(\widehat{\alpha}^{2 m+6}-\widehat{\beta}^{2 m+6}\right)-\left(\widehat{\alpha}^{2 m+3}-\widehat{\beta}^{2 m+3}\right)-\left(\widehat{\alpha}^{2 m+2}-\widehat{\beta}^{2 m+2}\right)}{\left(\widehat{\alpha}^{2 m+2}-\widehat{\beta}^{2 m+2}\right) v_{i-2}+\left(\widehat{\alpha}^{2 m+4}-\widehat{\beta}^{2 m+4}\right)-\left(\widehat{\alpha}^{2 m+1}-\widehat{\beta}^{2 m+1}\right)-\left(\widehat{\alpha}^{2 m}-\widehat{\beta}^{2 m}\right)}-2 \\
& =\frac{F_{2 m+4} v_{i-2}+F_{2 m+6}-F_{2 m+3}-F_{2 m+2}}{F_{2 m+2} v_{i-2}+F_{2 m+4}-F_{2 m+1}-F_{2 m}}-2 \\
& =\frac{F_{2 m+1} v_{i-2}+F_{2 m+2}}{F_{2 m+2} v_{i-2}+F_{2 m+3}},
\end{aligned}
$$

where $F_{n}$ is $n$-th Fibonacci number, $u_{i-2}=\frac{x_{i-2}}{y_{i-3}}, v_{i-2}=\frac{y_{i-2}}{x_{i-3}}$. From (2.1), (5.2), and (5.6), we get that, for $m \in \mathbb{N}_{0}$ and $i \in\{1,2\}$,

$$
\begin{aligned}
u_{2 m+1} & =\frac{F_{2 m+1} u_{-1}+F_{2 m+2}}{F_{2 m+2} u_{-1}+F_{2 m+3}} \\
& =\frac{x_{-1} F_{2 m+1}+y_{-2} F_{2 m+2}}{x_{-1} F_{2 m+2}+y_{-2} F_{2 m+3}}, \\
u_{2 m+2} & =\frac{F_{2 m+1} u_{0}+F_{2 m+2}}{F_{2 m+2} u_{0}+F_{2 m+3}} \\
& =\frac{y_{-1} F_{2 m+2}+x_{-2} F_{2 m+3}}{y_{-1} F_{2 m+3}+x_{-2} F_{2 m+4}} .
\end{aligned}
$$


Similarly, from (2.1), (5.2), and (5.7), we get that, for $m \in \mathbb{N}_{0}$ and $i \in\{1,2\}$,

$$
\begin{aligned}
v_{2 m+1} & =\frac{F_{2 m+1} v_{-1}+F_{2 m+2}}{F_{2 m+2} v_{-1}+F_{2 m+3}} \\
& =\frac{y_{-1} F_{2 m+1}+x_{-2} F_{2 m+2}}{y_{-1} F_{2 m+2}+x_{-2} F_{2 m+3}}, \\
v_{2 m+2} & =\frac{F_{2 m+1} v_{0}+F_{2 m+2}}{F_{2 m+2} v_{0}+F_{2 m+3}} \\
& =\frac{x_{-1} F_{2 m+2}+y_{-2} F_{2 m+3}}{x_{-1} F_{2 m+3}+y_{-2} F_{2 m+4}} .
\end{aligned}
$$

By substituting the formulas in (5.8)-(5.11) into (2.27)-(2.28) and changing indexes, we have the following results.

Theorem 5.1. Assume that $\left(x_{n}, y_{n}\right)_{n \geq-2}$ is a well-defined solution of the system (5.2). Then the following results are true.

$$
\begin{aligned}
& x_{2 m+1}=\frac{x_{-1} y_{-2}}{y_{-2} F_{2 m+3}+x_{-1} F_{2 m+2}}, \quad x_{2 m+2}=\frac{y_{-1} x_{-2}}{y_{-1} F_{2 m+3}+x_{-2} F_{2 m+4}}, \quad m \geq-1, \\
& y_{2 m+1}=\frac{y_{-1} x_{-2}}{x_{-2} F_{2 m+3}+y_{-1} F_{2 m+2}}, \quad y_{2 m+2}=\frac{x_{-1} y_{-2}}{x_{-1} F_{2 m+3}+y_{-2} F_{2 m+4}}, \quad m \geq-1, \\
& \text { where }\left\{F_{n}\right\}_{n=0}^{\infty}=\{0,1,1,2,3,5,8,13, \ldots\}, F_{-1}=1 .
\end{aligned}
$$

5.2. Case $b=-c=d=-\beta=-\gamma=\delta=1$

We will derive the solution forms of the system (5.1) with $b=-c=d=-\beta=-\gamma=$ $\delta=1$, that is, the system

$$
x_{n}=\frac{y_{n-1} x_{n-2}}{x_{n-2}-y_{n-1}}, y_{n}=\frac{x_{n-1} y_{n-2}}{-y_{n-2}-x_{n-1}}, n \in \mathbb{N}_{0},
$$

given in [41], through analytical approach. Also, the general solutions of the system (5.12) are expressed in terms of Fibonacci numbers. Now, to begin with, taking $b=-c=d=$ $-\beta=-\gamma=\delta=1$ in (2.12)-(2.13), we have

$$
q_{m+1}-q_{m}+\frac{1}{9} q_{m-1}=0, m \in \mathbb{N}_{0} .
$$

The characteristic equation of (5.13) can be clearly obtained as of the form $\lambda^{2}-\lambda+\frac{1}{9}=0$ with the roots $\lambda_{1}=\frac{3+\sqrt{5}}{6}=\frac{1}{3}\left(\frac{1+\sqrt{5}}{2}\right)^{2}=\frac{\widehat{\alpha}^{2}}{3}$ and $\lambda_{2}=\frac{3-\sqrt{5}}{6}=\frac{1}{3}\left(\frac{1-\sqrt{5}}{2}\right)^{2}=\frac{\widehat{\beta}^{2}}{3}$. On the other hand, taking into account $A=-3, B_{1}=-1, B_{2}=1, C_{1}=C_{2}=-2$ and the Binet Formula for Fibonacci numbers, then we can rewrite the equations in (2.18)-(2.19) as

$$
\begin{gathered}
u_{2 m+i}=3 \frac{\left(-\lambda_{1} u_{i-2}-2 \lambda_{1}+\frac{1}{3}\right) \lambda_{1}^{m+1}-\left(-\lambda_{2} u_{i-2}-2 \lambda_{2}+\frac{1}{3}\right) \lambda_{2}^{m+1}}{\left(-\lambda_{1} u_{i-2}-2 \lambda_{1}+\frac{1}{3}\right) \lambda_{1}^{m}-\left(-\lambda_{2} u_{i-2}-2 \lambda_{2}+\frac{1}{3}\right) \lambda_{2}^{m}}-2, \\
v_{2 m+i}=-3 \frac{\left(\lambda_{1} v_{i-2}-2 \lambda_{1}+\frac{1}{3}\right) \lambda_{1}^{m+1}-\left(\lambda_{2} v_{i-2}-2 \lambda_{2}+\frac{1}{3}\right) \lambda_{2}^{m+1}}{\left(\lambda_{1} v_{i-2}-2 \lambda_{1}+\frac{1}{3}\right) \lambda_{1}^{m}-\left(\lambda_{2} v_{i-2}-2 \lambda_{2}+\frac{1}{3}\right) \lambda_{2}^{m}}+2,
\end{gathered}
$$

for $m \in \mathbb{N}_{0}, i \in\{1,2\}$.

Using the relations $\widehat{\alpha} \widehat{\beta}=-1, \widehat{\alpha}^{2}+\widehat{\beta}=2, \widehat{\beta}^{2}+\widehat{\alpha}=2$ in (5.14)-(5.15) we get

$$
\begin{aligned}
u_{2 m+i} & =\frac{-\left(\widehat{\alpha}^{2 m+4}-\widehat{\beta}^{2 m+4}\right) u_{i-2}-\left(\widehat{\alpha}^{2 m+6}-\widehat{\beta}^{2 m+6}\right)+\left(\widehat{\alpha}^{2 m+3}-\widehat{\beta}^{2 m+3}\right)+\left(\widehat{\alpha}^{2 m+2}-\widehat{\beta}^{2 m+2}\right)}{-\left(\widehat{\alpha}^{2 m+2}-\widehat{\beta}^{2 m+2}\right) u_{i-2}-\left(\widehat{\alpha}^{2 m+4}-\widehat{\beta}^{2 m+4}\right)+\left(\widehat{\alpha}^{2 m+1}-\widehat{\beta}^{2 m+1}\right)+\left(\widehat{\alpha}^{2 m}-\widehat{\beta}^{2 m}\right)}-2 \\
& =\frac{-F_{2 m+4} u_{i-2}-F_{2 m+6}+F_{2 m+3}+F_{2 m+2}}{-F_{2 m+2} u_{i-2}-F_{2 m+4}+F_{2 m+1}+F_{2 m}}-2 \\
& =\frac{-F_{2 m+1} u_{i-2}-F_{2 m+2}}{-F_{2 m+2} u_{i-2}-F_{2 m+3}}
\end{aligned}
$$




$$
\begin{aligned}
v_{2 m+i} & =-\frac{\left(\widehat{\alpha}^{2 m+4}-\widehat{\beta}^{2 m+4}\right) v_{i-2}-\left(\widehat{\alpha}^{2 m+6}-\widehat{\beta}^{2 m+6}\right)+\left(\widehat{\alpha}^{2 m+3}-\widehat{\beta}^{2 m+3}\right)+\left(\widehat{\alpha}^{2 m+2}-\widehat{\beta}^{2 m+2}\right)}{\left(\widehat{\alpha}^{2 m+2}-\widehat{\beta}^{2 m+2}\right) v_{i-2}-\left(\widehat{\alpha}^{2 m+4}-\widehat{\beta}^{2 m+4}\right)+\left(\widehat{\alpha}^{2 m+1}-\widehat{\beta}^{2 m+1}\right)+\left(\widehat{\alpha}^{2 m}-\widehat{\beta}^{2 m}\right)}+2 \\
& =-\frac{F_{2 m+4} v_{i-2}-F_{2 m+6}+F_{2 m+3}+F_{2 m+2}}{F_{2 m+2} v_{i-2}-F_{2 m+4}+F_{2 m+1}+F_{2 m}}+2 \\
& =\frac{-F_{2 m+1} v_{i-2}+F_{2 m+2}}{F_{2 m+2} v_{i-2}-F_{2 m+3}}
\end{aligned}
$$

where $F_{n}$ is $n$-th Fibonacci number, $u_{i-2}=\frac{x_{i-2}}{y_{i-3}}, v_{i-2}=\frac{y_{i-2}}{x_{i-3}}$. From (2.1), (5.12), and (5.16), we get that, for $m \in \mathbb{N}_{0}$ and $i \in\{1,2\}$,

$$
\begin{aligned}
u_{2 m+1}= & \frac{-F_{2 m+1} u_{-1}-F_{2 m+2}}{-F_{2 m+2} u_{-1}-F_{2 m+3}} \\
= & \frac{-x_{-1} F_{2 m+1}-y_{-2} F_{2 m+2}}{-x_{-1} F_{2 m+2}-y_{-2} F_{2 m+3}}, \\
u_{2 m+2} & =\frac{-F_{2 m+1} u_{0}-F_{2 m+2}}{-F_{2 m+2} u_{0}-F_{2 m+3}} \\
& =\frac{y_{-1} F_{2 m+2}-x_{-2} F_{2 m+3}}{y_{-1} F_{2 m+3}-x_{-2} F_{2 m+4}} .
\end{aligned}
$$

Similarly, from (2.1), (5.12), and (5.17), we get that, for $m \in \mathbb{N}_{0}$ and $i \in\{1,2\}$,

$$
\begin{aligned}
v_{2 m+1} & =\frac{-F_{2 m+1} v_{-1}+F_{2 m+2}}{F_{2 m+2} v_{-1}-F_{2 m+3}} \\
& =\frac{-y_{-1} F_{2 m+1}+x_{-2} F_{2 m+2}}{y_{-1} F_{2 m+2}-x_{-2} F_{2 m+3}}, \\
v_{2 m+2} & =\frac{-F_{2 m+1} v_{0}+F_{2 m+2}}{F_{2 m+2} v_{0}-F_{2 m+3}} \\
& =\frac{-x_{-1} F_{2 m+2}-y_{-2} F_{2 m+3}}{x_{-1} F_{2 m+3}+y_{-2} F_{2 m+4}} .
\end{aligned}
$$

By substituting the formulas in (5.18)-(5.21) into (2.27)-(2.28) and changing indexes, we have the following results.

Theorem 5.2. Assume that $\left(x_{n}, y_{n}\right)_{n \geq-2}$ is a well-defined solution of the system (5.12). Then the following results are true.

$$
\begin{aligned}
& x_{2 m+1}=\frac{(-1)^{m+1} x_{-1} y_{-2}}{y_{-2} F_{2 m+3}+x_{-1} F_{2 m+2}}, \quad x_{2 m+2}=\frac{(-1)^{m+1} y_{-1} x_{-2}}{-y_{-1} F_{2 m+3}+x_{-2} F_{2 m+4}}, \quad m \geq-1, \\
& y_{2 m+1}=\frac{(-1)^{m+1} y_{-1} x_{-2}}{x_{-2} F_{2 m+3}-y_{-1} F_{2 m+2}}, \quad y_{2 m+2}=\frac{(-1)^{m} x_{-1} y_{-2}}{x_{-1} F_{2 m+3}+y_{-2} F_{2 m+4}}, \quad m \geq-1, \\
& \text { where }\left\{F_{n}\right\}_{n=0}^{\infty}=\{0,1,1,2,3,5,8,13, \ldots\}, F_{-1}=1 .
\end{aligned}
$$

5.3. Case $b=-c=d=\beta=\gamma=\delta=1$

We will derive the solution forms of the system (5.1) with $b=-c=d=\beta=\gamma=\delta=1$, that is, the system

$$
x_{n}=\frac{y_{n-1} x_{n-2}}{x_{n-2}-y_{n-1}}, y_{n}=\frac{x_{n-1} y_{n-2}}{y_{n-2}+x_{n-1}}, n \in \mathbb{N}_{0},
$$

given in [41], through analytical approach. Also, the general solutions of the system (5.22) are expressed in terms of Fibonacci numbers. Now, to begin with, taking $b=-c=d=$ $\beta=\gamma=\delta=1$ in (2.12)-(2.13), we have

$$
q_{m+1}-q_{m}-q_{m-1}=0, m \in \mathbb{N}_{0} .
$$


The characteristic equation of (5.23) can be clearly obtained as of the form $\lambda^{2}-\lambda-1=0$ with the roots $\lambda_{1}=\frac{1+\sqrt{5}}{2}=\widehat{\alpha}$ and $\lambda_{2}=\frac{1-\sqrt{5}}{2}=\widehat{\beta}$. On the other hand, taking into account $A=1, B_{1}=1, B_{2}=-1, C_{1}=0, C_{2}=2$ and the Binet Formula for Fibonacci numbers, we can rewrite the equations in (2.18)-(2.19) as for, $m \in \mathbb{N}_{0}$ and $i \in\{1,2\}$,

$$
\begin{gathered}
u_{2 m+i}=\frac{\left(\lambda_{1} u_{i-2}+1\right) \lambda_{1}^{m+1}-\left(\lambda_{2} u_{i-2}+1\right) \lambda_{2}^{m+1}}{\left(\lambda_{1} u_{i-2}+1\right) \lambda_{1}^{m}-\left(\lambda_{2} u_{i-2}+1\right) \lambda_{2}^{m}}, \\
v_{2 m+i}=-\frac{\left(-\lambda_{1} v_{i-2}+2 \lambda_{1}+1\right) \lambda_{1}^{m+1}-\left(-\lambda_{2} v_{i-2}+2 \lambda_{2}+1\right) \lambda_{2}^{m+1}}{\left(-\lambda_{1} v_{i-2}+2 \lambda_{1}+1\right) \lambda_{1}^{m}-\left(-\lambda_{2} v_{i-2}+2 \lambda_{2}+1\right) \lambda_{2}^{m}}+2,
\end{gathered}
$$

for $m \in \mathbb{N}_{0}, i \in\{1,2\}$.

Using the relations $\widehat{\alpha} \widehat{\beta}=-1, \widehat{\alpha}^{2}+\widehat{\beta}=2, \widehat{\beta}^{2}+\widehat{\alpha}=2$ in (5.24)-(5.25) we get

$$
\begin{aligned}
u_{2 m+i} & =\frac{\left(\widehat{\alpha}^{m+2}-\widehat{\beta}^{m+2}\right) u_{i-2}+\left(\widehat{\alpha}^{m+1}-\widehat{\beta}^{m+1}\right)}{\left(\widehat{\alpha}^{m+1}-\widehat{\beta}^{m+1}\right) u_{i-2}+\left(\widehat{\alpha}^{m}-\widehat{\beta}^{m}\right)} \\
& =\frac{F_{m+2} u_{i-2}+F_{m+1}}{F_{m+1} u_{i-2}+F_{m}}, \\
v_{2 m+i}= & \frac{\left(\widehat{\alpha}^{m+2}-\widehat{\beta}^{m+2}\right) v_{i-2}-\left(\widehat{\alpha}^{m+4}-\widehat{\beta}^{m+4}\right)}{-\left(\widehat{\alpha}^{m+1}-\widehat{\beta}^{m+1}\right) v_{i-2}+\left(\widehat{\alpha}^{m+3}-\widehat{\beta}^{m+3}\right)}+2 \\
= & \frac{F_{m+2} v_{i-2}-F_{m+4}}{-F_{m+1} v_{i-2}+F_{m+3}}+2 \\
= & \frac{-F_{m-1} v_{i-2}+F_{m+1}}{-F_{m+1} v_{i-2}+F_{m+3}},
\end{aligned}
$$

where $F_{n}$ is $n$-th Fibonacci number, $u_{i-2}=\frac{x_{i-2}}{y_{i-3}}, v_{i-2}=\frac{y_{i-2}}{x_{i-3}}$. From (2.1), (5.22) and (5.26), we get that, for $m \in \mathbb{N}_{0}$ and $i \in\{1,2\}$,

$$
\begin{aligned}
u_{2 m+1} & =\frac{F_{m+2} u_{-1}+F_{m+1}}{F_{m+1} u_{-1}+F_{m}} \\
& =\frac{x_{-1} F_{m+2}+y_{-2} F_{m+1}}{x_{-1} F_{m+1}+y_{-2} F_{m}}, \\
u_{2 m+2} & =\frac{F_{m+2} u_{0}+F_{m+1}}{F_{m+1} u_{0}+F_{m}} \\
& =\frac{-y_{-1} F_{m+1}+x_{-2} F_{m+3}}{-y_{-1} F_{m}+x_{-2} F_{m+2}} .
\end{aligned}
$$

Similarly, from (2.1), (5.22), and (5.27), we get that, for $m \in \mathbb{N}_{0}$ and $i \in\{1,2\}$,

$$
\begin{aligned}
v_{2 m+1} & =\frac{-F_{m-1} v_{-1}+F_{m+1}}{-F_{m+1} v_{-1}+F_{m+3}} \\
& =\frac{-y_{-1} F_{m-1}+x_{-2} F_{m+1}}{-y_{-1} F_{m+1}+x_{-2} F_{m+3}}, \\
v_{2 m+2} & =\frac{-F_{m-1} v_{0}+F_{m+1}}{-F_{m+1} v_{0}+F_{m+3}} \\
& =\frac{x_{-1} F_{m+1}+y_{-2} F_{m}}{x_{-1} F_{m+3}+y_{-2} F_{m+2}} .
\end{aligned}
$$

By substituting the formulas in (5.28)-(5.31) into (2.27)-(2.28) and changing indexes, we have the following results. 
Theorem 5.3. Assume that $\left(x_{n}, y_{n}\right)_{n \geq-2}$ is a well-defined solution of the system (5.22). Then the following results are true.

$$
\begin{gathered}
x_{2 m+1}=\frac{x_{-1} y_{-2}}{y_{-2} F_{m}+x_{-1} F_{m+1}}, \quad x_{2 m+2}=\frac{y_{-1} x_{-2}}{-y_{-1} F_{m}+x_{-2} F_{m+2}}, \quad m \geq-1, \\
y_{2 m+1}=\frac{y_{-1} x_{-2}}{x_{-2} F_{m+3}-y_{-1} F_{m+1}}, \quad y_{2 m+2}=\frac{x_{-1} y_{-2}}{x_{-1} F_{m+3}+y_{-2} F_{m+2}}, \quad m \geq-1,
\end{gathered}
$$

where $\left\{F_{n}\right\}_{n=0}^{\infty}=\{0,1,1,2,3,5,8,13, \ldots\}, F_{-1}=1$.

5.4. Case $b=c=d=\beta=-\gamma=\delta=1$

We will derive the solution forms of the system (5.1) with $b=c=d=\beta=-\gamma=\delta=1$, that is, the system

$$
x_{n}=\frac{y_{n-1} x_{n-2}}{x_{n-2}+y_{n-1}}, y_{n}=\frac{x_{n-1} y_{n-2}}{y_{n-2}-x_{n-1}}, n \in \mathbb{N}_{0},
$$

given in [41], through analytical approach. Also, the general solutions of the system (5.32) are expressed in terms of Fibonacci numbers. Now, to begin with, taking $b=c=d=\beta=$ $-\gamma=\delta=1$ in (2.12)-(2.13), we have

$$
q_{m+1}-q_{m}-q_{m-1}=0, m \in \mathbb{N}_{0} .
$$

The characteristic equation of (5.33) can be clearly obtained as of the form $\lambda^{2}-\lambda-1=0$ with the roots $\lambda_{1}=\frac{1+\sqrt{5}}{2}=\widehat{\alpha}$ and $\lambda_{2}=\frac{1-\sqrt{5}}{2}=\widehat{\beta}$. On the other hand, taking into account $A=1, B_{1}=-1, B_{2}=1, C_{1}=2, C_{2}=0$ and the Binet Formula for Fibonacci numbers, then we can rewrite the equations in (2.18)-(2.19) as

$$
\begin{gathered}
u_{2 m+i}=-\frac{\left(-\lambda_{1} u_{i-2}+2 \lambda_{1}+1\right) \lambda_{1}^{m+1}-\left(-\lambda_{2} u_{i-2}+2 \lambda_{2}+1\right) \lambda_{2}^{m+1}}{\left(-\lambda_{1} u_{i-2}+2 \lambda_{1}+1\right) \lambda_{1}^{m}-\left(-\lambda_{2} u_{i-2}+2 \lambda_{2}+1\right) \lambda_{2}^{m}}+2, \\
v_{2 m+i}=\frac{\left(\lambda_{1} v_{i-2}+1\right) \lambda_{1}^{m+1}-\left(\lambda_{2} v_{i-2}+1\right) \lambda_{2}^{m+1}}{\left(\lambda_{1} v_{i-2}+1\right) \lambda_{1}^{m}-\left(\lambda_{2} v_{i-2}+1\right) \lambda_{2}^{m}},
\end{gathered}
$$

for $m \in \mathbb{N}_{0}, i \in\{1,2\}$.

Using the relations $\widehat{\alpha} \widehat{\beta}=-1, \widehat{\alpha}^{2}+\widehat{\beta}=2, \widehat{\beta}^{2}+\widehat{\alpha}=2$ in (5.14)-(5.15) we get

$$
\begin{aligned}
u_{2 m+i}= & \frac{\left(\widehat{\alpha}^{m+2}-\widehat{\beta}^{m+2}\right) u_{i-2}-\left(\widehat{\alpha}^{m+4}-\widehat{\beta}^{m+4}\right)}{-\left(\widehat{\alpha}^{m+1}-\widehat{\beta}^{m+1}\right) u_{i-2}+\left(\widehat{\alpha}^{m+3}-\widehat{\beta}^{m+3}\right)}+2 \\
= & \frac{F_{m+2} u_{i-2}-F_{m+4}}{-F_{m+1} u_{i-2}+F_{m+3}}+2 \\
= & \frac{-F_{m-1} u_{i-2}+F_{m+1}}{-F_{m+1} u_{i-2}+F_{m+3}}, \\
v_{2 m+i} & =\frac{\left(\widehat{\alpha}^{m+2}-\widehat{\beta}^{m+2}\right) v_{i-2}+\left(\widehat{\alpha}^{m+1}-\widehat{\beta}^{m+1}\right)}{\left(\widehat{\alpha}^{m+1}-\widehat{\beta}^{m+1}\right) v_{i-2}+\left(\widehat{\alpha}^{m}-\widehat{\beta}^{m}\right)} \\
& =\frac{F_{m+2} v_{i-2}+F_{m+1}}{F_{m+1} v_{i-2}+F_{m}},
\end{aligned}
$$

where $F_{n}$ is $n$-th Fibonacci number, $u_{i-2}=\frac{x_{i-2}}{y_{i-3}}, v_{i-2}=\frac{y_{i-2}}{x_{i-3}}$. From (2.1), (5.32), and (5.36), we get that, for $m \in \mathbb{N}_{0}$ and $i \in\{1,2\}$,

$$
\begin{aligned}
u_{2 m+1} & =\frac{-F_{m-1} u_{-1}+F_{m+1}}{-F_{m+1} u_{-1}+F_{m+3}} \\
& =\frac{-x_{-1} F_{m-1}+y_{-2} F_{m+1}}{-x_{-1} F_{m+1}+y_{-2} F_{m+3}},
\end{aligned}
$$




$$
\begin{aligned}
u_{2 m+2} & =\frac{-F_{m-1} u_{0}+F_{m+1}}{-F_{m+1} u_{0}+F_{m+3}} \\
& =\frac{y_{-1} F_{m+1}+x_{-2} F_{m}}{y_{-1} F_{m+3}+x_{-2} F_{m+2}} .
\end{aligned}
$$

Similarly, from (2.1), (5.32), and (5.37), we get that, for $m \in \mathbb{N}_{0}$ and $i \in\{1,2\}$,

$$
\begin{aligned}
v_{2 m+1} & =\frac{F_{m+2} v_{-1}+F_{m+1}}{F_{m+1} v_{-1}+F_{m}} \\
& =\frac{y_{-1} F_{m+2}+x_{-2} F_{m+1}}{y_{-1} F_{m+1}+x_{-2} F_{m}}, \\
v_{2 m+2} & =\frac{F_{m+2} v_{0}+F_{m+1}}{F_{m+1} v_{0}+F_{m}} \\
& =\frac{-x_{-1} F_{m+1}+y_{-2} F_{m+3}}{-x_{-1} F_{m}+y_{-2} F_{m+2}} .
\end{aligned}
$$

By substituting the formulas in (5.38)-(5.41) into (2.27)-(2.28) and changing indexes, we have the following results.

Theorem 5.4. Assume that $\left(x_{n}, y_{n}\right)_{n \geq-2}$ is a well-defined solution of the system (5.32). Then the following results are true.

$$
\begin{gathered}
x_{2 m+1}=\frac{x_{-1} y_{-2}}{y_{-2} F_{m+3}-x_{-1} F_{m+1}}, \quad x_{2 m+2}=\frac{y_{-1} x_{-2}}{y_{-1} F_{m+3}+x_{-2} F_{m+2}}, \quad m \geq-1, \\
y_{2 m+1}=\frac{y_{-1} x_{-2}}{x_{-2} F_{m}+y_{-1} F_{m+1}}, \quad y_{2 m+2}=\frac{x_{-1} y_{-2}}{-x_{-1} F_{m}+y_{-2} F_{m+2}}, \quad m \geq-1, \\
\text { where }\left\{F_{n}\right\}_{n=0}^{\infty}=\{0,1,1,2,3,5,8,13, \ldots\}, F_{-1}=1 .
\end{gathered}
$$

Acknowledgment. The authors are thankful to the editor and reviewers for their constructive review.

\section{References}

[1] R.P. Agarwal, Difference Equations and Inequalities, New York USA, Marcel Dekker, 1992.

[2] R.P. Agarwal and E.M. Elsayed, On the solution of fourth-order rational recursive sequence, Adv. Stud. Contemp. Math. 20 (4), 525-545, 2010.

[3] I. Bajo and E. Liz, Global behaviour of a second-order nonlinear difference equation, J. Difference Equ. Appl. 17 (10), 1471-1486, 2011.

[4] L. Brand, A sequence defined by a difference equation, Am. Math. Mon. 62 (7), 489492, 1955.

[5] E. Camouzis and R. DeVault, The forbidden set of $x_{n+1}=p+\frac{x_{n-1}}{x_{n}}$, J. Difference Equ. Appl. 9 (8), 739-750, 2003.

[6] C. Cinar, On the positive solutions of difference equation, Appl. Math. Comput. 150 (1), 21-24, 2004.

[7] C. Cinar, S. Stevic, and I. Yalcinkaya, On positive solutions of a reciprocal difference equation with minimum, J. Appl. Math. Comput. 17 (1-2), 307-314, 2005.

[8] M. Dehghan, R. Mazrooei-Sebdani, and H. Sedaghat, Global behaviour of the Riccati difference equation of order two, J. Difference Equ. Appl. 17 (4), 467-477, 2011.

[9] I. Dekkar, N. Touafek, and Y. Yazlik, Global stability of a third-order nonlinear system of difference equations with period-two coefficients, Rev. R. Acad. Cienc. Exactas Fis. Nat. Ser. A Mat. 111 (2), 325-347, 2017. 
[10] Q. Din, M.N. Qureshi and A.Q. Khan, Dynamics of a fourth-order system of rational difference equations, Adv. Difference Equ. 2012 (215), 1-15, 2012.

[11] E.M. Elabbasy, H.A. El-Metwally, and E.M. Elsayed, Global behavior of the solutions of some difference equations, Adv. Difference Equ. 2011 (1), 1-16, 2011.

[12] M.E. Elmetwally and E.M. Elsayed, Dynamics of a rational difference equation, Chin. Ann. Math. Ser. B 30 (2), 187-198, (2009).

[13] E.M. Elsayed, Qualitative behavior of a rational recursive sequence, Indag. Math. 19 (2), 189-201, 2008.

[14] E.M. Elsayed, Qualitative properties for a fourth order rational difference equation, Acta Appl. Math. 110 (2), 589-604, 2010.

[15] E.A. Grove and G. Ladas, Periodicities In Nonlinear Difference Equations, Chapman \& Hall, CRC Press, Boca Raton, 2005.

[16] N. Haddad, N. Touafek, and J.F.T. Rabago, Solution form of a higher-order system of difference equations and dynamical behavior of its special case, Math. Methods Appl. Sci. 40 (10), 3599-3607, 2017.

[17] N. Haddad, N. Touafek, and J.F.T. Rabago, Well-defined solutions of a system of difference equations, J. Appl. Math. Comput. 56 (1-2), 439-458, 2018.

[18] Y. Halim, N. Touafek, and Y. Yazlik, Dynamic behavior of a second-order nonlinear rational difference equation, Turkish J. Math. 39 (6), 1004-1018, 2015.

[19] T. Koshy, Fibonacci and Lucas Numbers with Applications, Wiley, New York (2001).

[20] M.R.S. Kulenovic and O. Merino, Discrete Dynamical Systems and Difference Equations with Mathematica, New York, NY, USA: CRC Press, 2002.

[21] L.C. McGrath and C. Teixeira, Existence and behavior of solutions of the rational equation $x_{n+1}=\frac{a x_{n-1}+b x_{n}}{c x_{n-1}+d x_{n}} x_{n}$, Rocky Mountain J. Math. 36 (2), 649-674, 2006.

[22] I. Okumus and Y. Soykan, Dynamical behavior of a system of three-dimensional nonlinear difference equations, Adv. Difference Equ. 2018 (223), 1-15, 2018.

[23] Ö. Öcalan, Oscillation of nonlinear difference equations with several coefficients, Commun. Math. Anal. 4 (1), 35-44, 2008.

[24] Ö. Öcalan and O. Akin, Oscillation properties for advanced difference equations, Novi Sad J. Math. 37 (1), 39-47, 2007.

[25] G. Papaschinopoulos and C.J. Schinas, On a system of two difference equations, J. Math. Anal. Appl. 219 (2), 415-426, 1998.

[26] G. Papaschinopoulos and G. Stefanidou, Asymptotic behavior of the solutions of a class of rational difference equations, Int. J. Difference Equ. 5 (2), 233-249, 2010.

[27] A. Raouf, Global behaviour of the rational riccati difference equation of order two: the general case, J. Difference Equ. Appl. 18 (6), 947-961, 2012.

[28] A. Raouf, Global behavior of the higher order rational riccati difference equation, Appl. Math. Comput. 230, 1-8, 2014.

[29] J. Rubió-Massegú, On the existence of solutions for difference equations, J. Difference Equ. Appl. 13 (7), 655-664, 2007.

[30] H. Sedaghat, Existence of solutions for certain singular difference equations, J. Difference Equ. Appl. 6 (5), 535-561, 2000.

[31] H. Sedaghat, Global behaviours of rational difference equations of orders two and three with quadratic terms, J. Difference Equ. Appl. 15 (3), 215-224, 2009.

[32] S. Stević, On some solvable systems of difference equations, Appl. Math. Comput. 218 (9), 5010-5018, 2012.

[33] S. Stević, M.A. Alghamdi, N. Shahzad, and D.A. Maturi, On a class of solvable difference equations, Abstr. Appl. Anal. 2013, 1-7, 2013.

[34] S. Stević, J. Diblík, B. Iričanin, and Z. Šmarda, On a solvable system of rational difference equations, J. Difference Equ. Appl. 20 (5-6), 811-825, 2014. 
[35] J. Sugie, Nonoscillation theorems for second-order linear difference equations- via the riccati-type transformation II, Appl. Math. Comput. 304, 142-152, 2017.

[36] N. Taskara, K. Uslu, and D.T. Tollu, The periodicity and solutions of the rational difference equation with periodic coefficients, Comput. Math. Appl. 62 (4), 1807-1813, 2011.

[37] D.T. Tollu, Y. Yazlik, and N. Taskara, On the solutions of two special types of riccati difference equation via Fibonacci numbers, Adv. Difference Equ. 1 (2013), 1-7, 2013.

[38] D.T. Tollu, Y. Yazlik, and N. Taskara, On fourteen solvable systems of difference equations, Appl. Math. Comput. 233, 310-319, 2014.

[39] D.T. Tollu, Y. Yazlik, and N. Taskara, On a solvable nonlinear difference equation of higher order, Turkish J. Math. 42 (4), 1765-1778, 2018.

[40] N. Touafek, On a second order rational difference equation, Hacet. J. Math. Stat. 41 (6), 867-874, 2012.

[41] N. Touafek and E.M. Elsayed, On a second order rational systems of difference equations, Hokkaido Math. J. 44 (1), 29-45, 2015.

[42] I. Yalcinkaya, On the difference equation $x_{n+1}=\alpha+\frac{x_{n-m}}{x_{n}^{k}}$, Discrete Dyn. Nat. Soc. 2008, 1-8, 2008.

[43] X. Yang, On the system of rational difference equations $x_{n}=A+\frac{y_{n-1}}{x_{n-p} y_{n-q}}, y_{n}=$ $A+\frac{x_{n-1}}{x_{n-r} y_{n-s}}$, J. Math. Anal. Appl. 307, 305-311, 2006.

[44] Y. Yazlik, On the solutions and behavior of rational difference equations, J. Comput. Anal. Appl. 17 (3), 584-594, 2014.

[45] Y. Yazlik, E.M. Elsayed, and N. Taskara, On the behaviour of the solutions of difference equation systems, J. Comput. Anal. Appl. 16 (5), 932-941, 2014.

[46] Y. Yazlik, D.T. Tollu, and N. Taskara, On the behaviour of solutions for some systems of difference equations, J. Comput. Anal. Appl. 18 (1), 166-178, 2015.

[47] Y. Yazlik, D.T. Tollu, and N. Taskara, On the solutions of a max-type difference equation system, Math. Methods Appl. Sci. 38 (17), 4388-4410, 2015.

[48] Y. Yazlik, D.T. Tollu, and N. Taskara, On the solutions of a three-dimensional system of difference equations, Kuwait J. Sci. 43 (1), 95-111, 2016. 